\title{
phénomènes cycliques dans les sols pulvérulents
}

\author{
par
}

\section{Luong Minh Phong}

Laboratoire de Mécanique des Solides - Ecole Polytechnique

\section{Introduction}

Le rapide développement du Génie Civil dans des domaines géotechniques en liaison avec des effets cycliques, transitoires et dynamiques dus aux éléments naturels (houle, courants, vents,...), aux actions séismiques, ou à des technologies spéciales (vibrofonçage, machines tournantes,...) a suscité un intérêt particulièrement suivi pour les aspects cycliques du comportement mécanique des sols pulvérulents.

\begin{abstract}
Les principaux résultats d'essais effectués sur des sables très différents (un sable de Fontainebleau, un sable de Loire, un sable carbonaté de la Manche, figure 1), au moyen d'essais triaxiaux classiques sur des échantillons cylindriques, seront présentés ici. Le fait que deux contraintes principales soient égales entraine une restriction inhérente à la technique expérimentale choisie. L'appareil triaxial permet néanmoins d'explorer le comportement mécanique du sol suivant des trajets de charge assez variés et de mettre en évidence des propriétés caractéristiques intéressantes, en particulier dans le cas des sollicitations répétées.
\end{abstract}

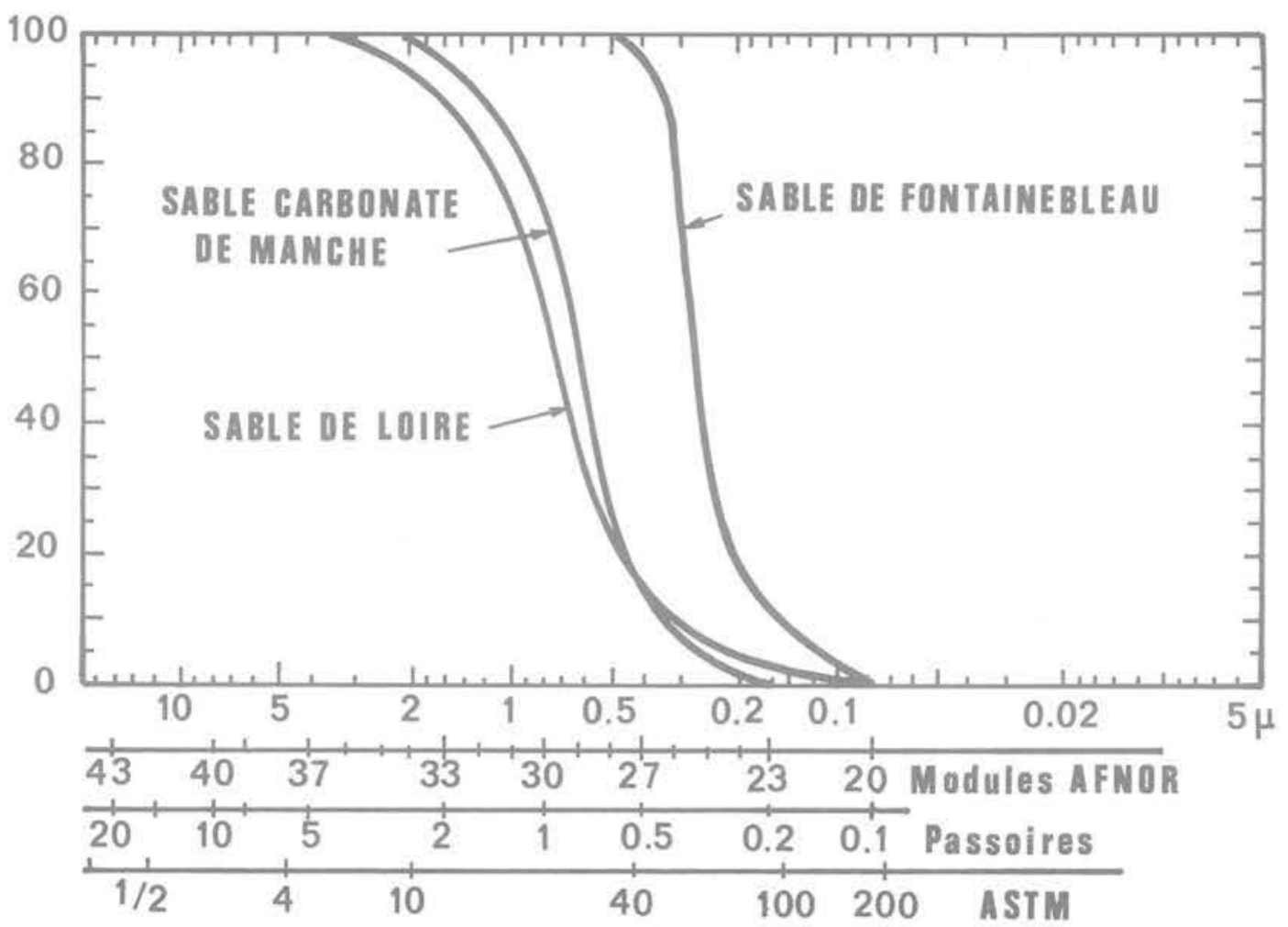

Fig. 1 Courbes granulométriques 


\section{Notations des paramètres adoptés}

L'échantillon cylindrique est repéré géométriquement par son axe longitudinal (direction 1) et sa section droite circulaire (directions 2 et 3 perpendiculaires et équivalentes). Les paramètres de chargement adoptés sont respective-

- la contrainte moyenne $\quad \mathrm{p}=\frac{\sigma_{1}+2 \sigma_{3}}{3}$

- la contrainte déviatorique $q=\sigma_{1}-\sigma_{3}$

- le niveau des contraintes $\eta=\frac{q}{p}$

Les paramètres de déformation sont :

- la déformation volumétrique

$$
\begin{aligned}
& \epsilon_{\mathrm{V}}=\epsilon_{1}+2 \epsilon_{3} \\
& \epsilon_{\mathrm{q}}=\frac{2}{3}\left(\epsilon_{1}-\epsilon_{3}\right) \\
& \beta=\frac{\epsilon_{\mathrm{q}}}{\epsilon_{\mathrm{V}}}
\end{aligned}
$$$$
\text { - le niveau distorsionnel }
$$

Les essais ont été réalisés en compression triaxiale (raccourcissement axial) et en extension triaxiale (allongement axial) à vitesse de charge $\sigma_{1}$ constante ou à vitesse de déformation $\hat{\epsilon}_{1}$ constante en condition saturée, drainée ou non drainée.

Avec la notation $\sigma_{1} \geqslant \sigma_{2} \geqslant \sigma_{3}$, nous notons une rotation du trièdre des contraintes principales entre les états de compression et d'extension triaxiale. Les chemins de contrainte explorés sont donc localisés dans des plans méridiens de symétrie ternaire du trièdre des contraintes.

Les conditions opératoires imposées à l'échantillon d'élancement $H / D \geqslant 2$ sont, soit des conditions non drainées, soit des conditions drainées, et dans un tel cas on peut distinguer:

1- $\sigma_{2}=\sigma_{3}=\mathrm{cte}$

c'est le cas de l'essai triaxial conventionnel à pression de confinement constante ;

II - $\mathrm{p}=$ cte

la contrainte moyenne est maintenue constante en faisant varier la pression de confinement avec la charge axiale :

III $-\mathrm{q}=$ cte

la contrainte déviatorique sur l'échantillon reste constante au cours du chargement :

IV - $\eta=$ cte

sur ce chemin radial dans l'espace des contraintes

l'obliquité moyenne des contraintes est constante :

$\mathrm{V}-\sigma=\left(\sigma_{1}^{2}+\sigma_{2}^{2}+\sigma_{3}^{2}\right)^{1 / 2}=\mathrm{cte}$

ou $\rho=\left(p^{2}+q^{2}\right)^{1 / 2}=$ cte

cette condition correspond à des trajets de charge sphérique ou circulaire respectivement dans l'espace des contraintes principales $\left(\sigma_{1}, \sigma_{2}, \sigma_{3}\right)$ et dans le diagramme $(p, q)$; l'obliquité moyenne des contraintes varie au cours de ce chargement à module de contrainte constant :

$\mathrm{VI}-\epsilon_{\mathrm{y}}=0$

l'essai est effectué à volume global de l'échantillon constant.

\section{Constatations expérimentales}

Les déformations du sol sous charge sont essentiellement les conséquences de trois mécanismes

1 - la compressibilité et les changements de forme du squelette solide :

II - les glissements et les rotations des particules liés à l'obliquité des contraintes et associés à une réorientation des grains, pouvant créer une anisotropie de structure :

III - la rupture et l'attrition des grains élémentaires modifiant la texture du matériau.

Les variations volumétriques résultent principalement de deux mécanismes :

I - une contraction ou une dilatation générale du sque- lette solide sans modification del'architecture général des grains :

II - des variations de l'enchevêtrement des grains. Elles sont surtout sensibles dans les essais de cisaillement, mais elles peuvent aussi se produire lors du premier chargement hydrostatique d'un sable lâche.

La distorsion dépend de l'arrangement particulier des particules solides présentant ou non des possibilités de blocage ou de déblocage.

Le serrage, le desserrage et le désenchevêtrement sont des mécanismes qui entraînent des déformations irréversibles importantes. Par contre, la contraction et la dilatation traduisent des déformations plus limitées quasi-réversibles, le nombre des points de contact entre grains restant sensiblement constant au cours des variations des contraintes.

\subsection{Compression triaxiale monotone}

A partir d'un état de contrainte isotrope de confinement maintenu constant, en augmentant la contrainte déviatorique $\mathrm{q}=\sigma_{1}-\sigma_{3}$, on observe une déformation volumétrique qui est d'abord une contraction, puis qui ralentit, s'annule dans le cas d'un sable lâche ou s'inverse avec un sable dense pour permettre un désenchevêtrement des grains avant les grands déplacements relatifs. Le rapport - $d \epsilon_{3} / d \epsilon_{1}$ de la déformation latérale à la déformation axiale, d'abord négligeable, croît régulièrement de zéro à une valeur dépassant largement la valeur 0,5. Le foisonnement constaté, appelé dilatance, est d'autant plus important que l'arrangement initial du sable était compact (fig. 2). II diminue lorsque la pression de confinement augmente.

\subsection{Extension triaxiale}

En diminuant algébriquement la contrainte déviatorique $q$ on réalise très aisément un essai triaxial cylindrique en extension (fig. 3). La contrainte principale $\sigma_{2}$ intermédiaire, au lieu d'être égale à la contrainte principale mineure dans le cas précédent, a la même valeur que la contrainte principale majeure. On constate une contraction de volume malgré une diminution de la contrainte moyenne p suivie comme dans le cas précédent d'une dilatance. Cette variation du volume initial dans le sens inverse de la contrainte moyenne $p$ appliquée, montre que le coefficient - $d \epsilon_{3} / d \epsilon_{1}$ est initialement plus grand que 0,5 . Aucune réversibilité, même approchée, n'est évidemment possible pour ce trajet de premier chargement.

\subsection{Comparaison de ces deux types d'essai}

L'analogie de l'évolution de la déformation volumétrique masque de grandes différences de comportement rhéologique entre deux types d'essais :

1 - la déformabilité du sol est plus grande en compression triaxiale; le matériau apparaît plus raide en extension triaxiale :

II - le coefficient de Poisson - $d \epsilon_{3} / d \epsilon_{1}$ au début de chargement est plus faible en compression $(<0,5)$ qu'en extension triaxiale $(>0,5)$;

III - la résístance au cisaillement au seuil du désenchevêtrement des grains, selon la représentation de Mohr. est plus importante en compression qu'en extension triaxiale $\left(\varphi_{\mathrm{CT}}>\varphi_{\mathrm{ET}}\right)$ avec les sables étudiés : sable de Fontainebleau, sable de Loire, sable carbonaté de Manche :

IV - le trajet de charge dans le diagramme $p, q$ lors d'un essai couplé de compression-extension triaxiales suit une droite de pente 3 : la compression triaxiale correspond à la partie positive de q et l'extension triaxialeà la partie négative. En réalité, il y a eu changement de plan méridien par rapport à l'axe des contraintes moyennes, lors du passage à l'état $q=0$;

$\mathrm{V}$ - le désenchevêtrement à partir du seuil caractéristique en compression triaxiale se traduit par une dilatance importante en volume. En extension triaxiale, on constate au contraire des déformations distorsionnelles prépondérantes pour une dilatance relativement plus limitée. 

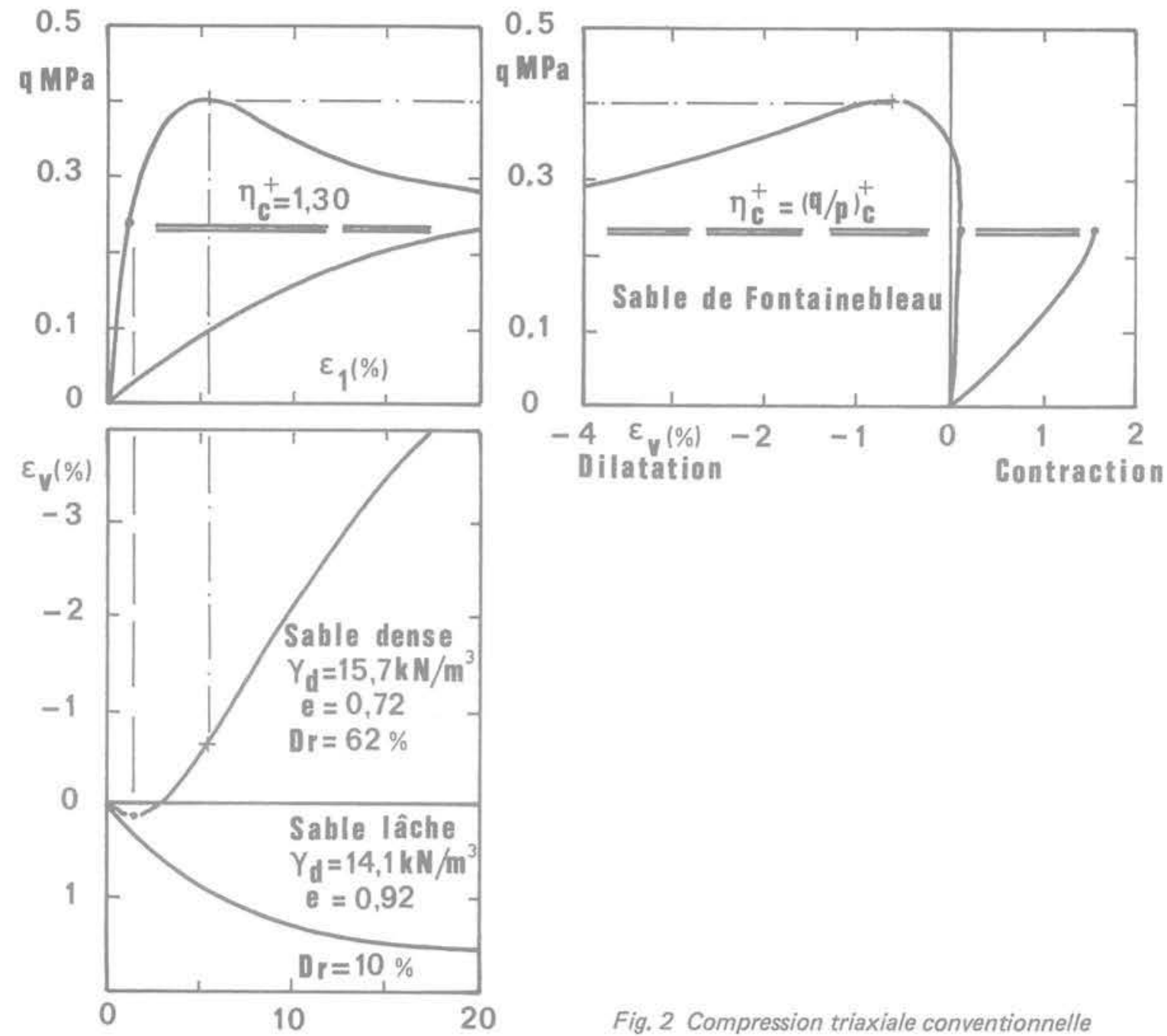

Fig. 2 Compression triaxiale conventionnelle
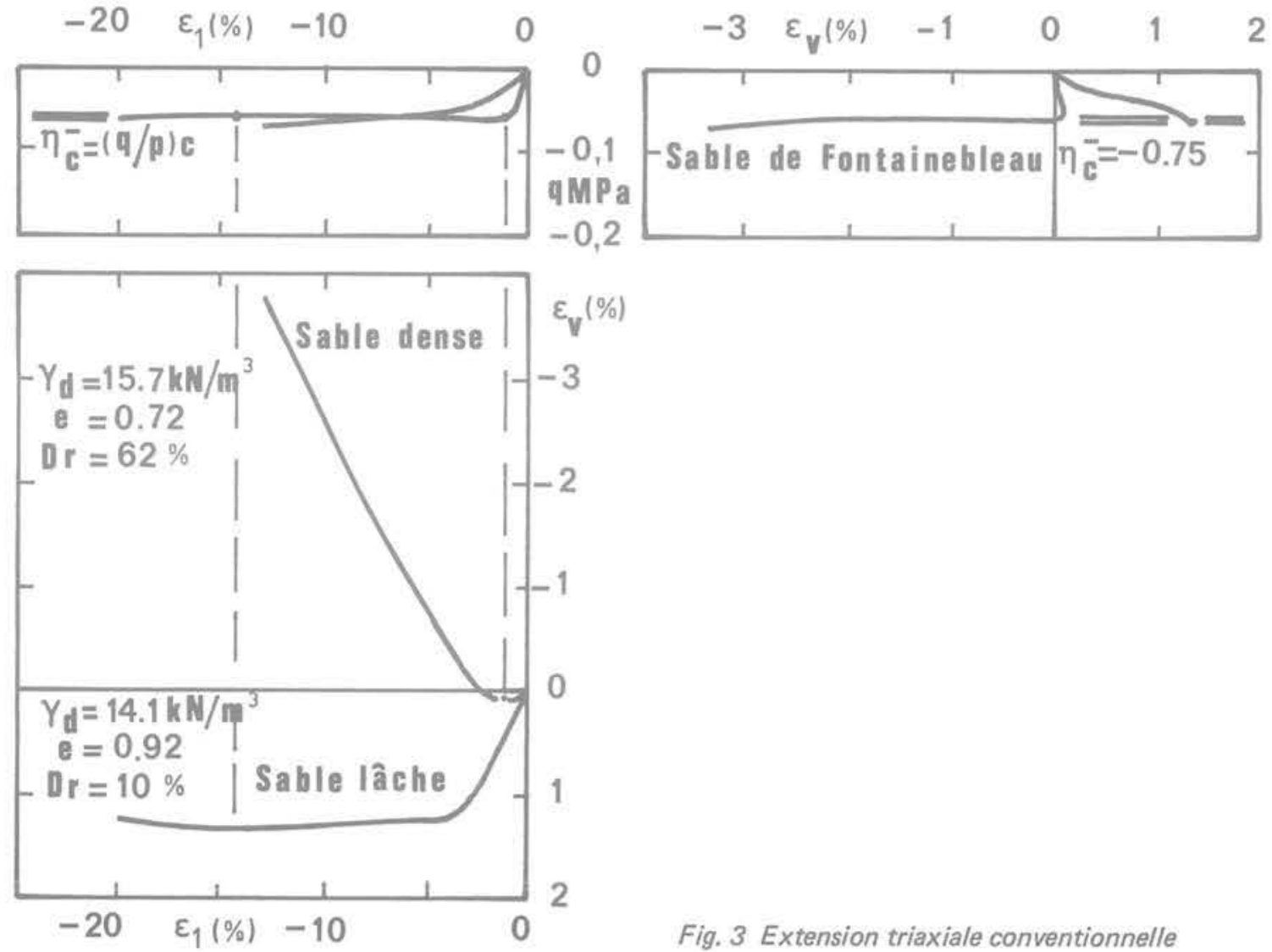

Fig. 3 Extension triaxiale conventionnelle 


\section{Concept de l'état caractéristique}

Si la déformation est suivie dès le début du chargement, on peut facilement constater les trois points principaux suivants :

1 - la différence de comportement entre le premier chargement et les cycles suivants de décharge-recharge traduisant l'effet du serrage initial :

II - l'existence des phènomènes d'hystérésis plus ou moins importants suivant le trajet de charge indiquant l'établissement d'un mode d'arrangement ou l'apparition du désenchevêtrement des grains :

III - I'importance relative des variations de volume par rapport aux déformations de distorsion provenant du couplage entre la compressibilité volumétrique et la distorsion par glissement des grains.

L'étude des sols sous chargement non monotone nécessite donc un concept nouveau pour pouvoir suivre le comportement rhéologique tout au long du processus de la déformation irréversible dans la phase transitoire précédant les seuils admissibles en contraintes ou en déformations.

\subsection{Concept actuel : état critique}

L'analogie du comportement mécanique du métal et des sols est mise en évidence sur le diagramme contraintedéformation (fig. 4), où $\sigma_{\mathrm{E}}$ est le seuil d'écoulement du matériau. L'existence d'un palier horizontal a suggéré le concept d'un état idéalisé du sol, appelé Etat Critique, associè à un volume spécifique $v$, et défini par

$$
q=M p \quad \text { et } \quad v=\Gamma-\lambda L n p
$$

oủ $\mathrm{M}, \Gamma, \lambda$ sont des constantes caractérisant le matériau (Schofield et Wroth, 1968).

Ce concept très fécond a permis de réaliser des progrès significatifs dans la compréhension et dans l'élaboration des techniques de calcul en mécanique des sols sous chargement statique monotone jusqu'à la charge ultime.

L'état critique traduit un comportement asymptotique du sol dans le domaine des grandes déformations à la rupture oú l'écoulement se produit sans variation de volume, mais seulement lorsque l'indice des vides $e=v-1$ est à un niveau critique $e_{c r}$.ll ne permet donc pas de suivre la déformation irréversible dès le début, et s'avère inadéquat à l'étude du comportement des sols sous sollicitations non monotones.

\subsection{Concept nouveau : état caractéristique}

Les essais triaxiaux conventionnels en condition drainée -en compression $\left(\sigma_{1}>\sigma_{2}=\sigma_{3}\right)$ comme en extension $\left(\sigma_{1}<\sigma_{2}=\sigma_{3}\right)$ - permettent de définir de faccon très simple par le niveau de contrainte correspondant au passage de la déformation volumétrique dilatante, un êtat caractéristique du sol qui, compatible avec l'état, est associé à :

1 - un taux de variation de volume nul $\epsilon_{\mathrm{y}}=0$;

II - un seuil de changement de comportement pour ce trajet de charge ; le processus d'enchevêtrement des grains se termine pour laisser la place au désenchevêtrement. L'angle $\varphi_{c}$ associé à ce seuil et donné par la relation $\frac{\sigma_{t}}{\sigma_{3}}=\operatorname{tg}^{2}\left(\frac{\pi}{4}+\frac{\varphi_{c}}{2}\right)$ caractérise la capacité d'enchevêtrement du matériau (Kirkpatrick, 1961). L'angle caractéristique $\varphi_{C}$ est un facteur intrinsèque du matériau :

III - un domaine de déformations du sol relativement faibles et stables bien avant la rupture ;

IV - une indépendance vis à vis de la porosité initiale:

$V$ - une non-influence de l'anisotropie des contacts et des chaînons de grains :

VI - une insensibilitẻ à la granulométrie, (Habib, Luong, 1978).

Pour ce trajet de charge, en condition drainée à pression de confinement constante, l'état caractéristique sépare dans le domaine des contraintes admissibles deux types de comportement théologique du sol: contractant dans le domaine subcaractéristique délimité dans le plan $(p, q)$ par les lignes caractéristiques LC, (fig. 5), et dilatant dans le domaine sur-

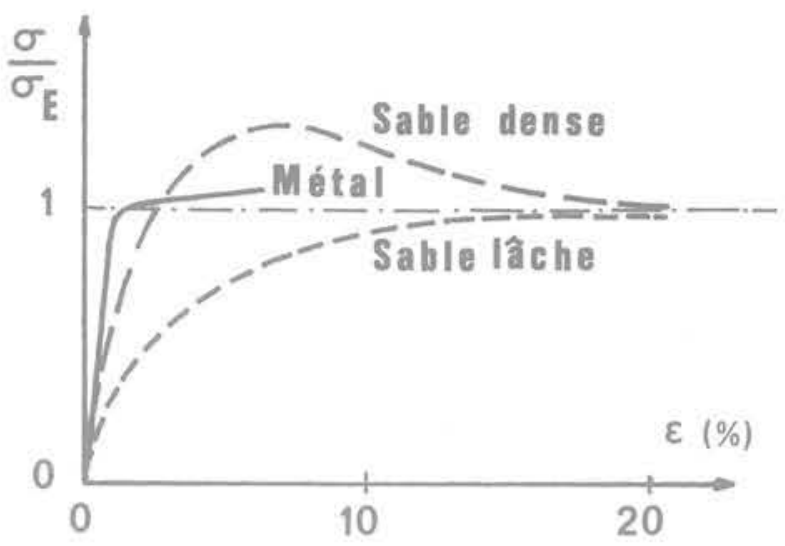

Fig. 4 Analogie métal-sol

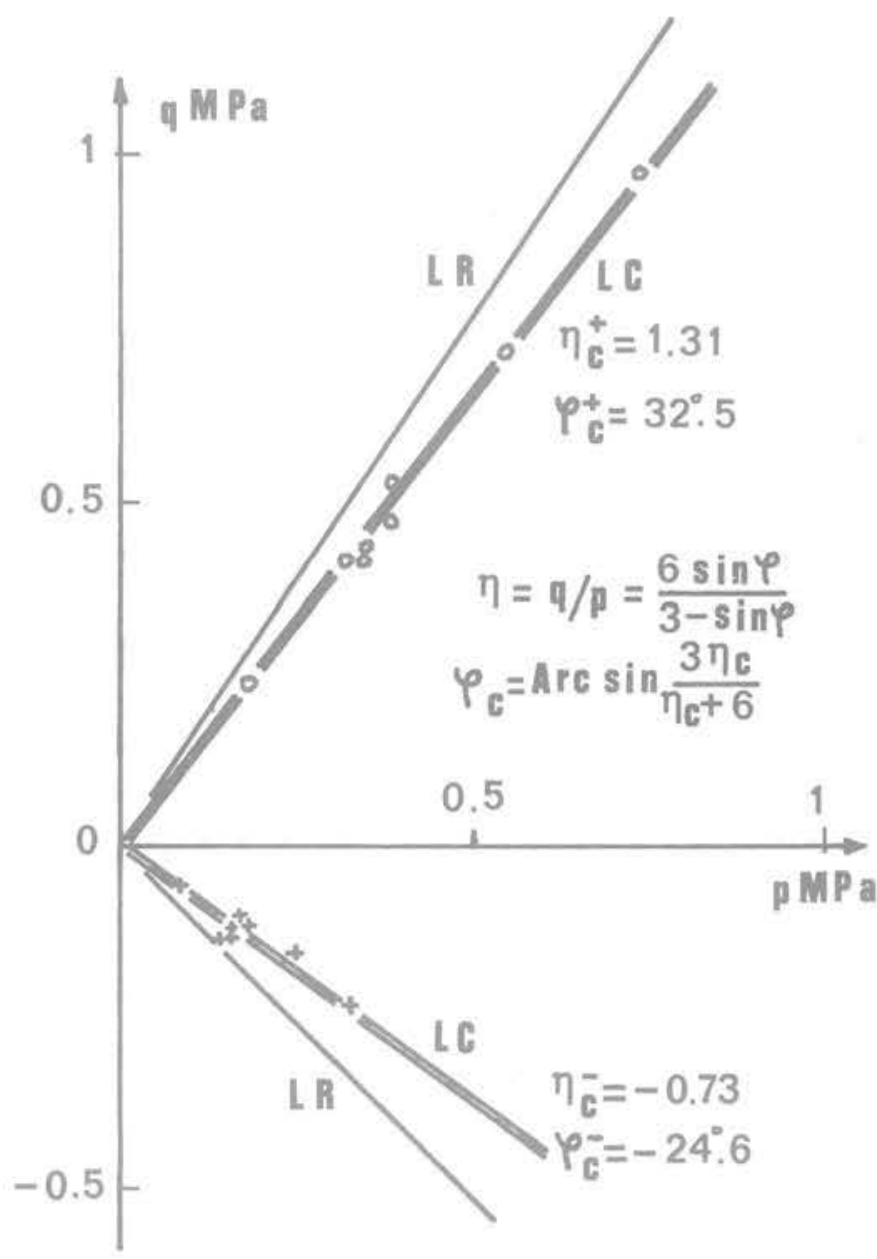

Fig. 5 Critère caractéristique

(le double trait représente le seuil caractéristique)

caractéristique jusqu'à la limite de rupture LR, existant ou non, comme c'est le cas des sables très lâches. Dans ce dernier cas, l'état caractéristique s'identifie à l'état critique. Mis en évidence de manière très simple sur le trajet triaxial conventionnel à pression de confinement constante, l'état caractéristique se confirme-t-il encore comme un seuil de désenchevêtrement de la structure granulaire lorsque le chargement suit d'autres trajets dans le plan $(p, q)$ ? 
Pression atmosphérique

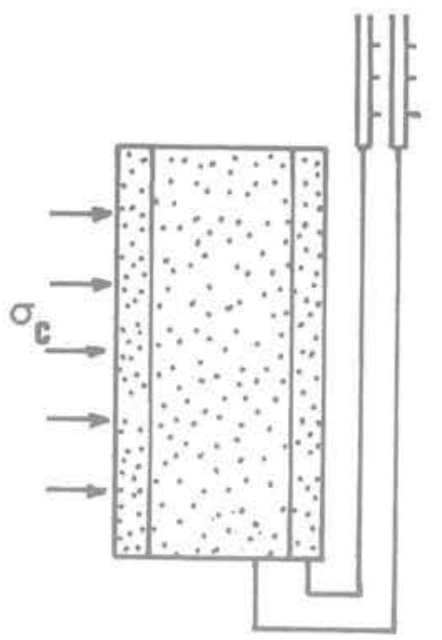

Tubes de mesure des variations de volume

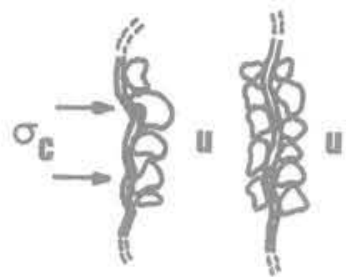

Fig. 6 Eprouvette à confinement variable
Les essais présentés ci-après ont été conduits à pression de confinement variable. L'effet de la pénétration de la membrane peut induire des erreurs appréciables dans la mesure des variations de volume. II a été nécessaire de réaliser le montage expérimental de la figure 6 pour pouvoir minimiser cet effet ou évaluer l'étalonnage et la précision relative des mesures de déformations volumétriques.

D'une façon générale, les différents résultats expérimentaux montrent que, quel que soit le chemin de contrainte suivi, l'état caractéristiques se définit par la valeur du rapport $d \epsilon_{\mathrm{v}} / \mathrm{d} \epsilon_{\mathrm{q}}$ diminuant brusquement vers une faible valeur pouvant être nulle, c'est-à-dire que le niveau distorsionnel $\beta$ augmente sensiblement entrainant à la fois la dilatance par désenchevêtrement et une distorsion considérable du squelette granulaire du sol.

\subsection{Cisaillement drainé à contrainte moyenne $p$ constante}

Les essais en condition drainée à contrainte moyenne p constante à l'appareil triaxial de révolution donnent des résultats analogues à ceux obtenus sur le trajet rectiligne de pente 3 dans le diagramme $(p, q)$.

Les figures 7 et 8 représentent respectivement un essai de compression triaxiale et un essai en extension triaxiale à $p=c t e$. Les seuils caractéristiques déterminés sur les courbes $q, \epsilon_{\mathrm{v}}$ à $\mathrm{p}=$ cte concordent bien avec les valeurs trouvées suivant les trajets à pression de confinement constante.

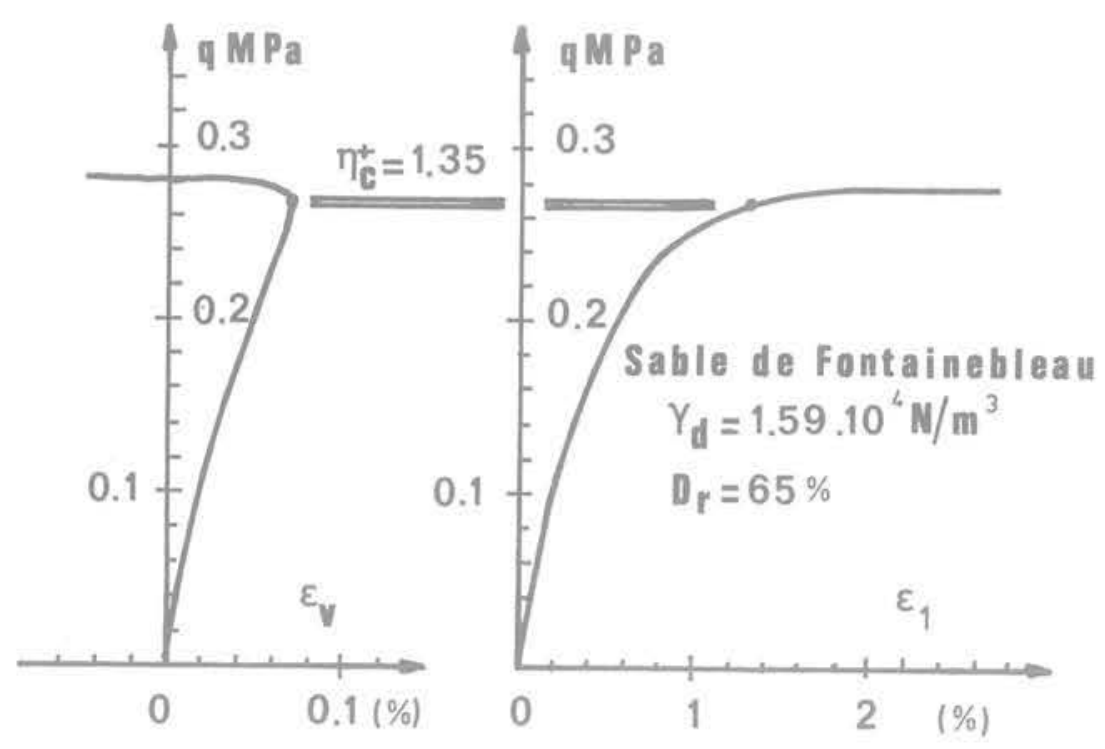

Fig. 7 Compression triaxiale $A p=0.20 \mathrm{MPa}=$ Cte (le double trait représente le seuil caractéristique)

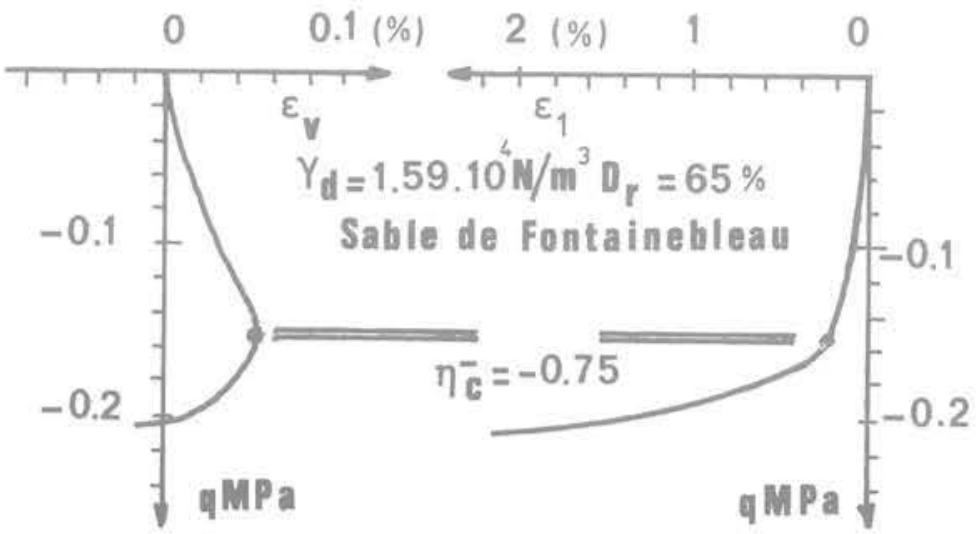

Fig. 8 Extension triaxiale à $p=0.20 \mathrm{MPa}=$ Cte (le double trait représente le seuil caractéristique) 


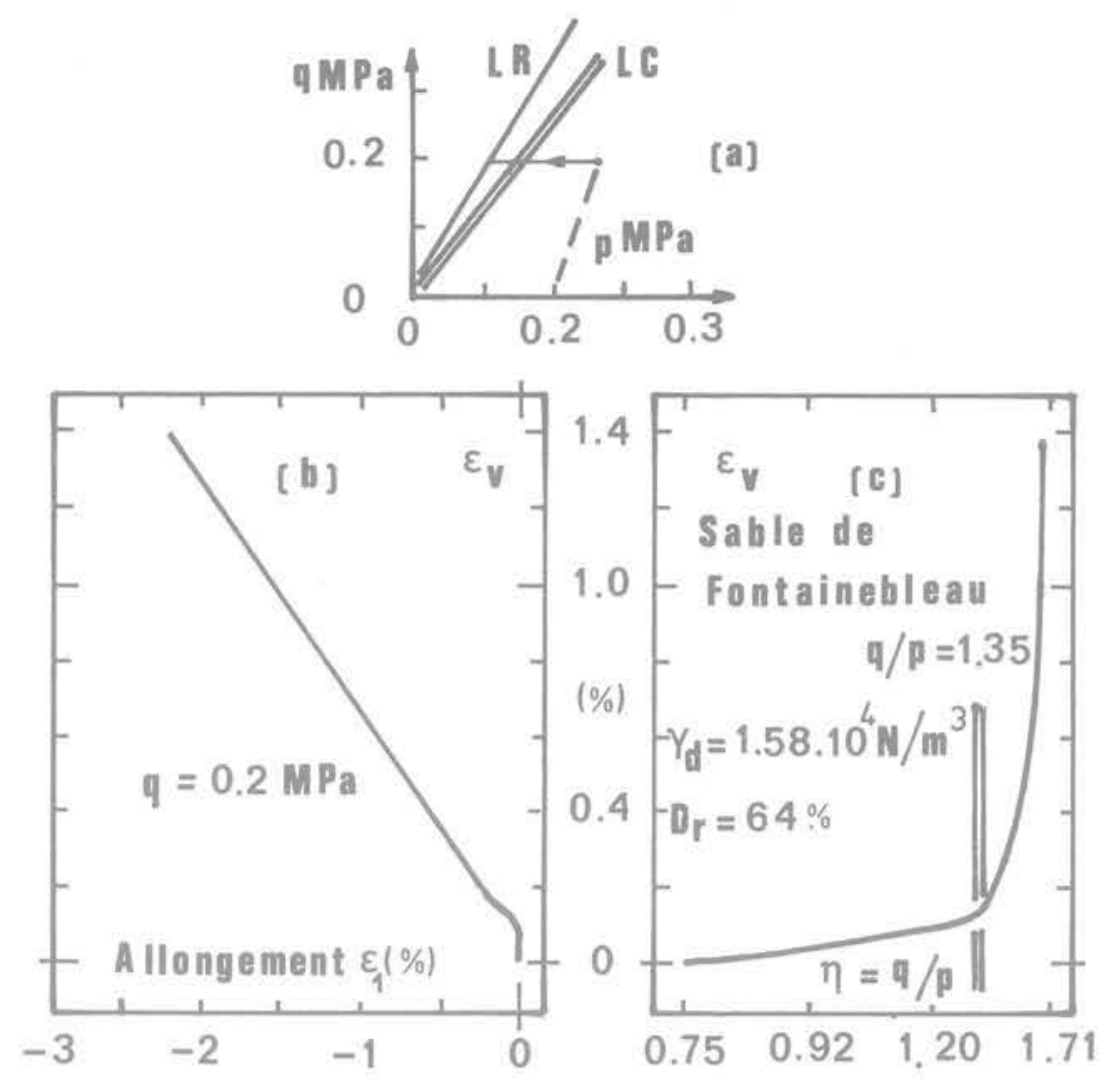

Fig. 9 Chargement drainé à déviateur constant

\subsection{Chargement drainé à déviateur q constant}

A partir d'une pression de confinement constante $\sigma_{1}=\sigma_{3}=0,2 \mathrm{MPa}$, on augmente progressivement la contrainte déviatorique $\mathrm{q}=\sigma_{1}-\sigma_{3}$ jusqu'à la valeur $0,2 \mathrm{MPa}$. Sous ce déviateur maintenu constant, on diminue ensuite la pression de confinement pour réaliser un essai drainé à déviateur constant.

La figure 9.a montre, par une ligne fléchée, le trajet de charge dans le diagramme $(p, q)$. la figure 9.6 donne la variation de la déformation volumétrique $\epsilon_{\mathrm{y}}$ en fonction de l'allongement $\epsilon_{1}$. A l'intérieur de la frontiẻre caractéristique $L C, \epsilon_{1}$ varie très peu avec $\epsilon_{1}$. Dès que le seuil caractéristique est atteint, la distorsion augmente rapidement avec la déformation volumétrique. La figure 9.c décrit l'évolution de $\epsilon_{y}$ avec le niveau de contrainte $\eta=q / p$. Dés que $\eta$ atteint le niveau caractéristique $\eta_{C}$, la déformation volumétrique s'amplifie pour traduire le phénomène de dilatance.

\subsection{Caractéristiques contraintes-déformations sous trajet de charge radial en condition drainée}

Les trajets de charge à rapport $q / p=\eta$ constant sont des chemins radiaux dans l'espace des contraintes: l'obliquité moyenne de la contrainte appliquée sur une facette - ainsi que celle des forces de contacts intergranulaires - est maintenue constante au cours du chargement (EI-Sohby, 1969).

1 - Sous contrainte isotrope oủ $\eta=0$ (fig. 10), les caractéristiques contraintes-déformations dépendent de la densité initiale et de la pression de confinement (ou de la pression moyenne au cours du chargement). Plus le matériau est lâche, plus la variation de volume est grande, et plus cette variation contient une grande partie irréversible lors du premier chargement. Ceci est dû au mécanisme de serrage.

II - Pour les valeurs de rl inférieures au seuil d'enchevêtrement $\eta_{c}$ correspondant à l'état caractéristique, le changement de géométrie de la structure des grains est relativement faible. Le sol se contracte en s'enche-
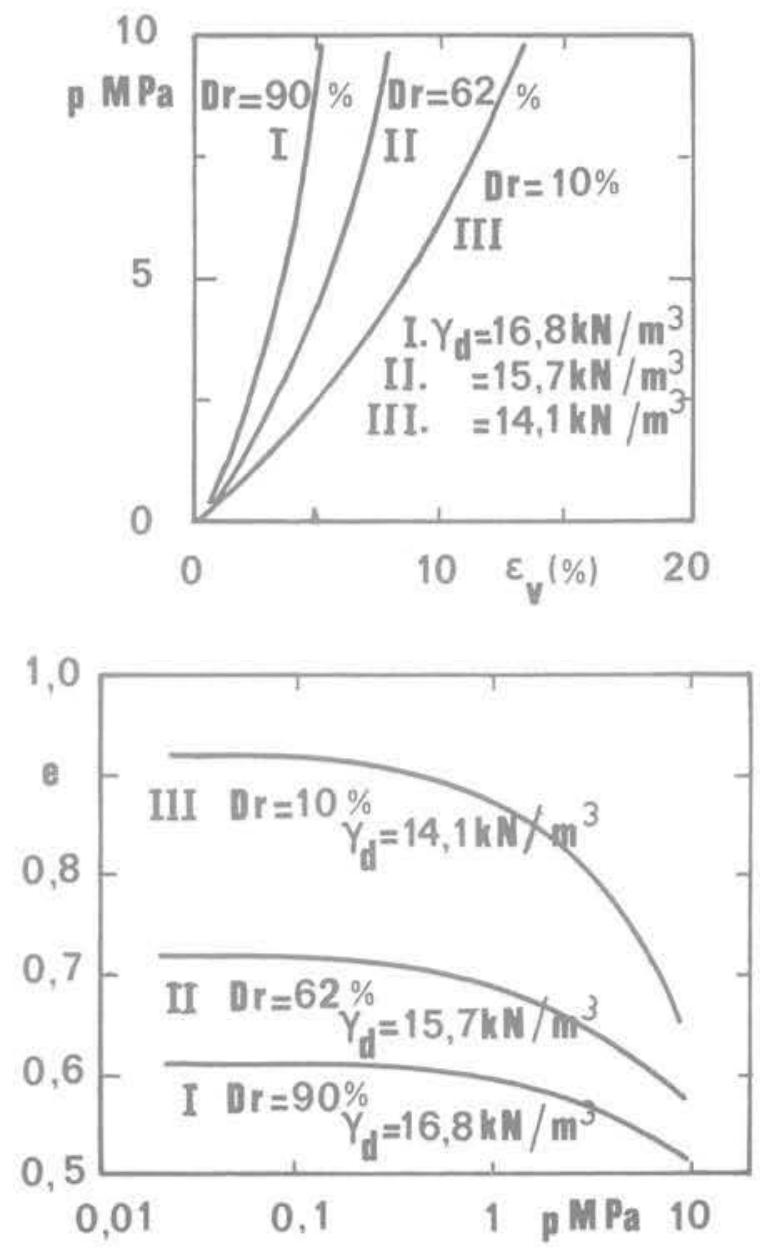

Fig. 10 Compressibilité du sable de Fontainebleau sous contrainte isotrope 


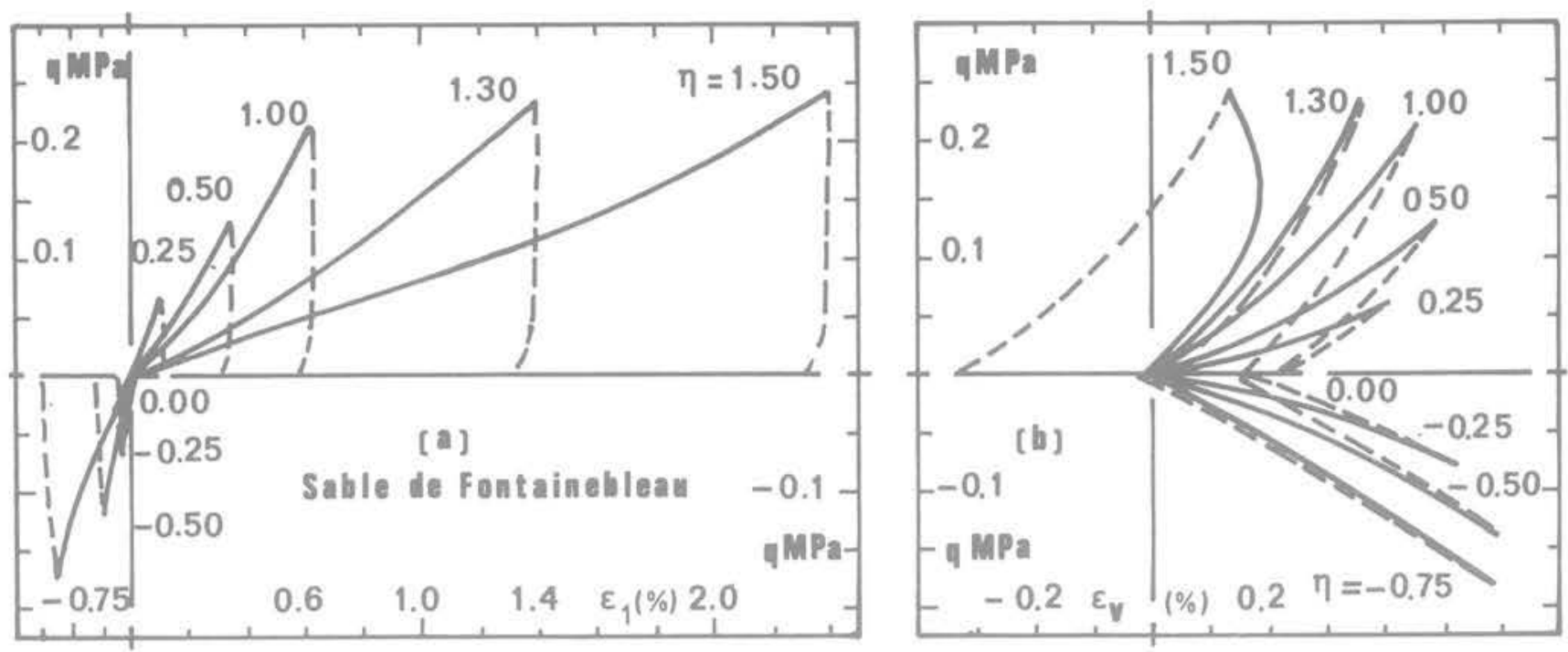

Fig. 11 Chargement radial en condition drainée (compression et extension triaxiales)
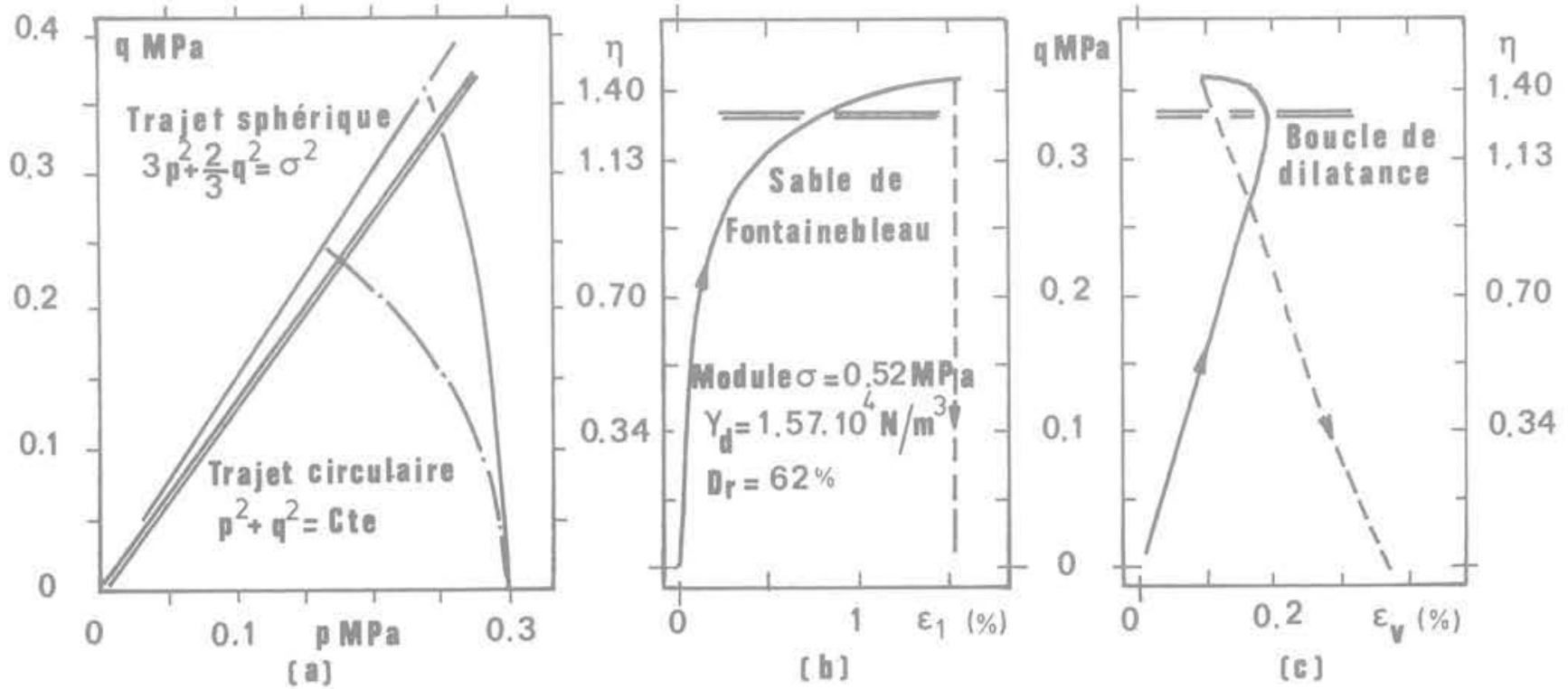

Fig. 12 Cisaillement drainé sur un trajet sphérique

vêtrant lors de la charge et se dilate par relâchement des forces de contact au cours de la décharge (fig. 11 a et b).

III - Lorsque le rapport $\eta$ dépasse la valeur $\eta_{C}$ du seuil caractéristique, un grand nombre de particules se mettent à glisser à cause de la forte obliquité moyenne des forces de contact intergranulaire ou des contraintes sur leur facette. On constate aisément un phénomène de dilatance et de foisonnement aussi bien en charge qu'en décharge. La dilatance volumique est prépondérante dans le cas de l'essai en compression triaxiale : par contre, le cas en extension triaxiale est surtout marqué par des déformations distortionnelles irréversibles très importantes.

Ce type d'essais à trajet radial de charge met clairement en évidence l'importance du concept de l'état caractéristique qui délimite un domaine subcaractéristique des contacts intergranulaires stables. Au-delà, dans le domaine surcaractéristique, la forte obliquité des forces de contacts intergranulaires facilite des glissements de forte amplitude entraînant le désenchevêtrement de la structure des grains, ou une distorsion importante de la structure.

\subsection{Chargement drainé à obliquité de contrainte variable}

Le chargement radial maintient constante l'obliquité moyenne de la contrainte. Par contre, le trajet sphérique dans l'espace des contraintes principales $\left(\sigma_{1}, \sigma_{2}, \sigma_{3}\right)$ ou circulaire dans le diagramme ( $p, q)$ de l'essai triaxial (fig. 12a) permet de caractériser l'influence de l'inclinaison de la contrainte sur le comportement rhéologique du matériau en fonction du module de contrainte maintenu constant au cours du chargement.

\section{I - Trajet sphérique}

Les figures 12.b et $12 . \mathrm{c}$ montrent respectivement l'évolution de $\epsilon_{1}$ et $\epsilon_{\mathrm{y}}$ en fontion du déviateur q ou du niveau de contrainte $\eta=q / p$. Sur le diagramme $\left(q, \epsilon_{y}\right)$, on n'observe pas de boucle d'hystérésis à la recharge si le seuil caractéristique n'a pas été atteint. Lorsqu'il est dépassé, une boucle de dilatance apparaît distinctement lors de la décharge (fig. 12.c). 

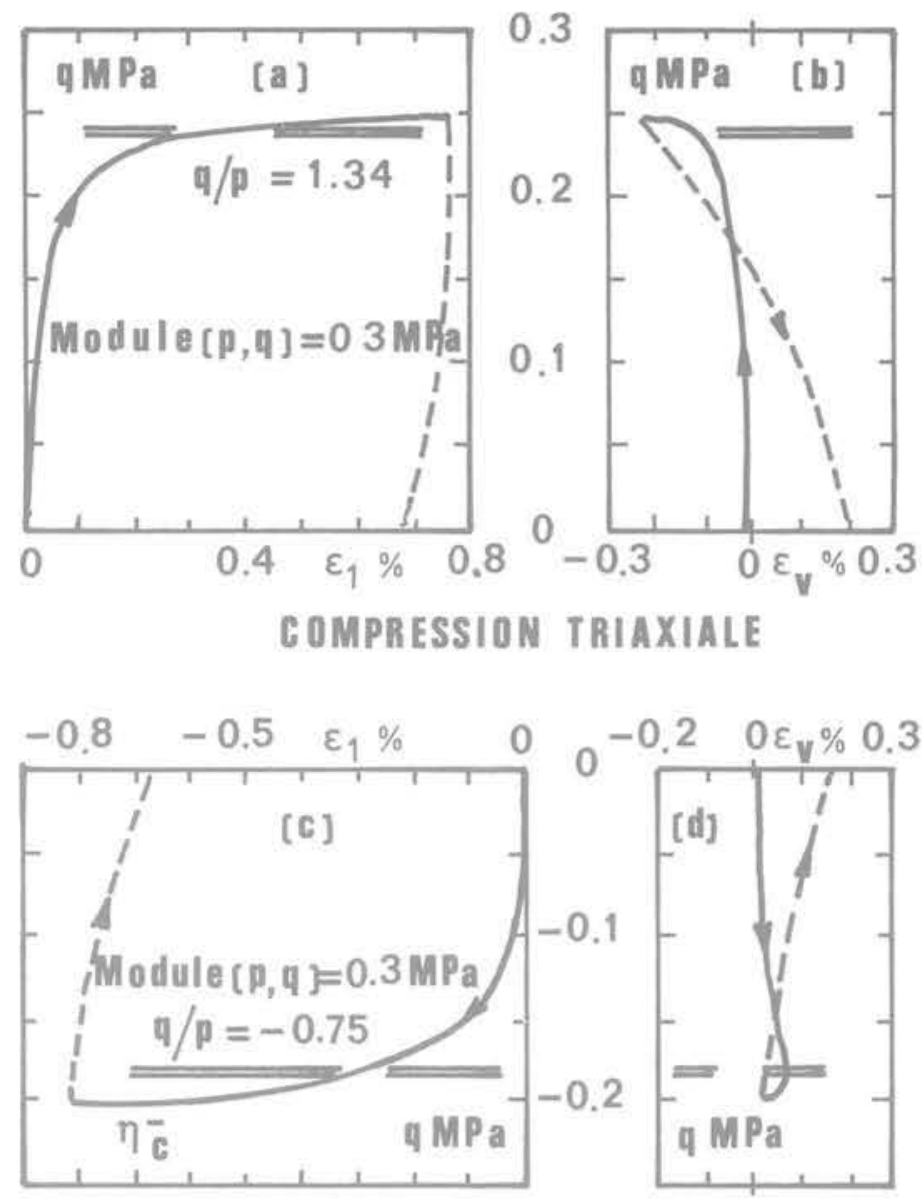

EXTENSION TRIAXIALE

Fig. 13 Cisaillement drainé à trajet circulaire dans le diagramme $(p, q)$

\section{II - Trajet circulaire}

Les figures 13.a.b.c.d. indiquent respectivement l'allure de la variation des déformations axiale $\epsilon_{1}$ et volumétrique $\sigma_{y}$ avec q en compression triaxiale et en extension triaxiale lors d'un essai de cisaillement en condition drainée à module $(p, q)$ constant.

D'une manière analogue au cas sphérique, on n'observe pas de boucle d'hystérésis pour la variation de volume dans le domaine subcaractéristique. La boucle de dilatance n'apparaît que lorsque le seuil caractéristique est franchi.

Dans les deux cas, sphérique ou circulaire, on peut constater facilement que lorsque le seuil caractéristique $\eta_{c}$ est atteint, le niveau distorsionnel $\beta$ augmente brusquement indiquant le début d'une phase de désenchevêtrement de la structure granulaire. La décharge fait apparaître une contraction volumétrique $\epsilon_{\mathrm{v}}$ appréciable, alors que la déformation axiale $\epsilon_{1}$ reste beaucoup plus limitée.

L'état caractéristique peut être repéré suivant ces deux trajets de charge par l'existence ou non d'une boucle de dilatance lors de la décharge.

\subsection{Trajet de charge lors d'un essai drainé à volume constant}

Ce type d'essai préconisé par Taylor (1948) offre une repré- sentation correcte du comportement des grandes masses de sables saturés cisaillés suffisamment rapidement pour qu'il n'y ait pas drainage de l'eau interstitielle.

Les figures 14.a.b.c. montrent les relations contraintesdéformations $\left(q, \epsilon_{1}\right)$ et les chemins des contraintes $(p, q)$ des essais à volume constant en compression et en extension triaxiales. Lorsque le trajet de charge atteint le seuil caractéristique en compression comme en extension, il s'incurve pour remonter la ligne caractéristique confirmant ainsi que l'état caractéristique constitue bien un seuil d'enchevêtrement au-delà duquel de grands glissements dus à la distorsion - c'est-à-dire le désenchevêtrement des grains peuvent se manifester.

Expérimentalement, ces essais en compression et extension triaxiales à volume constant permettent de préciser la direction des incréments de charge du plan $(p, q)$ correspondant à un relâchement de la structure granulaire sans désenchevêtrement, (faible augmentation de volume), ou à une contraction par enchevêtrement plus important du milieu granulaire, (diminution de volume). On constate une déformabilité à la décharge d'autant plus importante que le trajet suit plus longtemps la ligne caractéristique. Quelques cycles de charges atteignant le seuil caractéristique et de décharge peuvent conduire le point figuratif de la contrainte vers l'origine $O$ du plan $(p, q)$. Ce qui signifierait une perte totale de la résistance. 


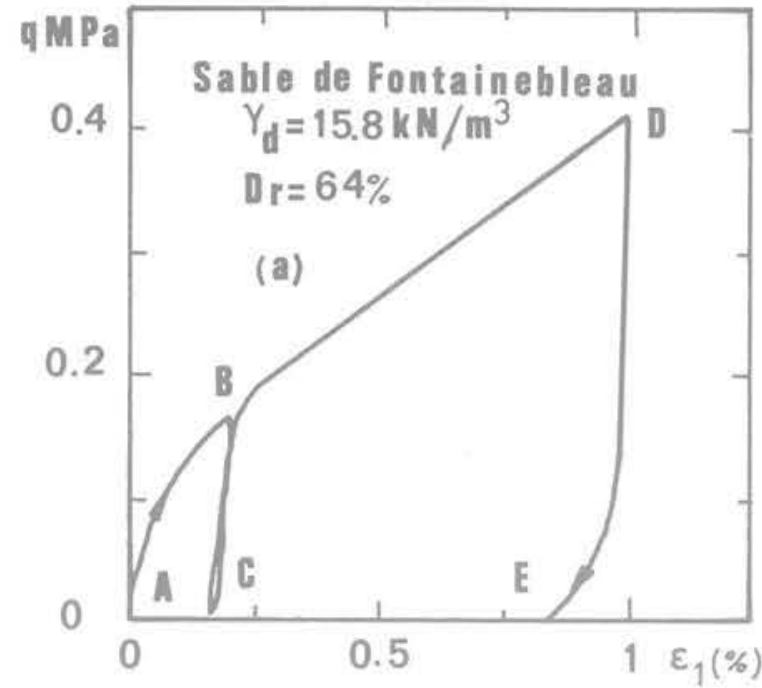

Fig. 14 a Compression triaxiale à volume constant

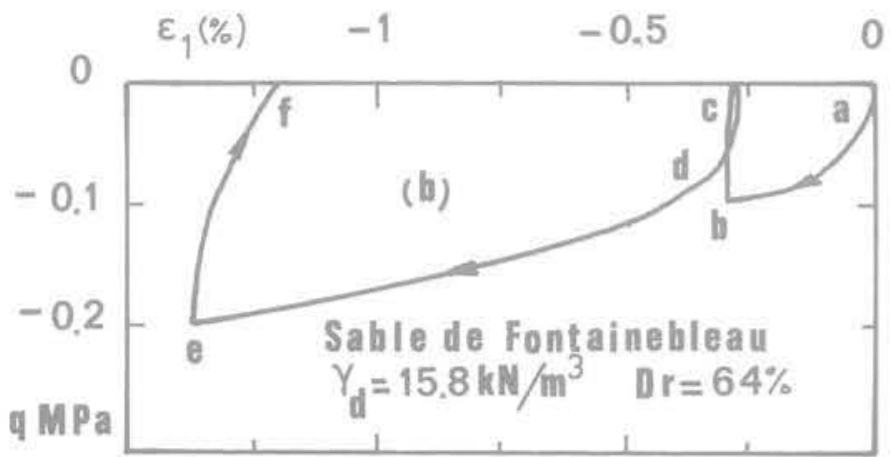

Fig. 14 b Extension triaxiale à volume constant

\subsection{Trajet de contrainte effective en condition non-drainée}

Lorsqu'on effectue un essai triaxial conventionnel en condition non drainée, on constate (fig. 15) une augmentation de pression interstitielle u traduisant une tendance à la contraction du squelette solide. Cette pression des pores, mesurée au cours du chargement suivant le trajet de contrainte totale T.C.T., détermine le trajet de contrainte effective T.C.E. dans le plan (p, q). Les cycles de chargedécharge mettent en évidence l'écrouissage du sol.

Dès que le seuil caractéristique est atteint, l'expérience montre que le trajet de contrainte effective suit la ligne caractéristique L.C. La suppression interstitielle u diminue au fur et à mesure que le squelette du sol tend à se dilater. Lors de la décharge, on observe une déformabilité d'autant plus grande que le chemin parcouru par le point figuratif de la contrainte effective sur la ligne caractéristique est plus long. La recharge suivante montre un comportement de sable vierge associé à son nouvel état initial, car la structure des grains a été détruite durant le parcours du point figuratif de la contrainte effective sur la ligne caractéristique.

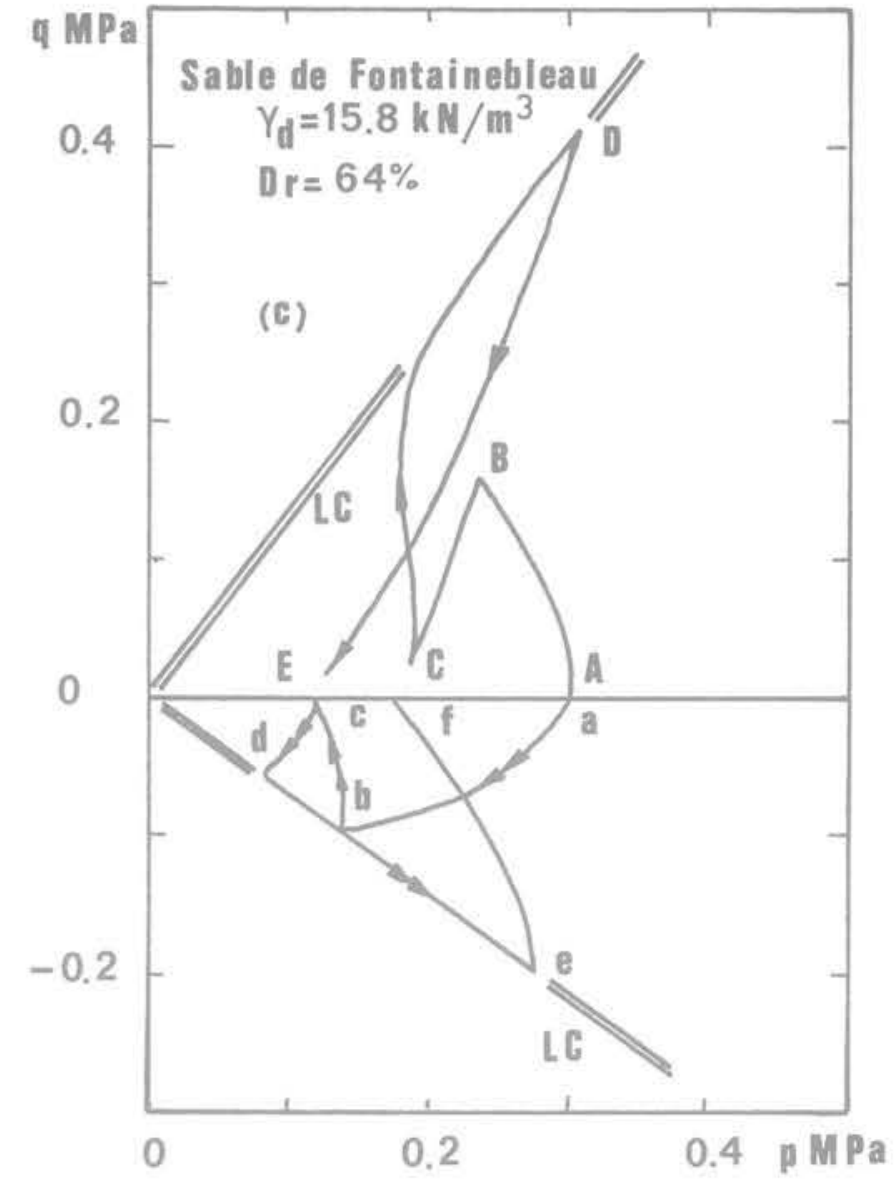

Fig. $14 \mathrm{C}$ Chemins de contrainte à volume constant

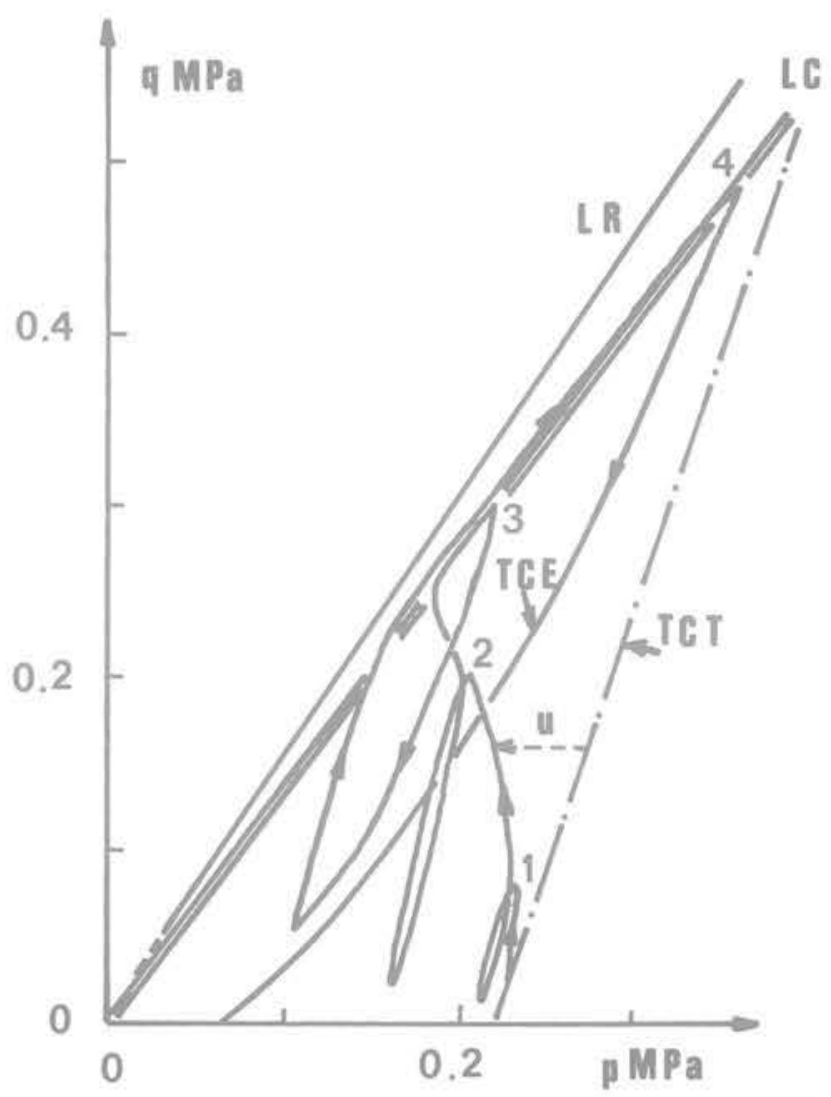

Fig. 15 Trajet de contrainte effective TCE en condition non drainée 


\section{Comportements cycliques des sols pulvérulents}

L'état caractéristique s'est révélé un concept cohérent pour l'étude des divers comportements cycliques des sols sableux.

\subsection{Déformations sous des cycles de chargements radiaux}

La figure 16 montre la déformation volumétrique totale $\epsilon_{\mathrm{y}}$ et la partie $\epsilon \mathrm{p}_{\mathrm{V}}^{\mathrm{p}}$ sous chargement hydrostatique $(\eta=0)$ en condition drainée après un cycle, après dix cycles, et enfin après un grand nombre de cycles de façon à atteindre la valeur asymptotique de $\epsilon \underset{y}{\mathrm{P}}$. Le sol présente alors un comportement quasi-élastique non linéaire (Ko et Scott, 1967). L'adaptation peut être considérée comme obtenue pour un nombre fini de cycles de chargement isotrope.

Sous chargements radiaux de rapports $\eta=\frac{q}{p}$ différents de zéro, mais inférieurs en valeur absolue aux seuils caractéristiques en compression et extension triaxiales, le sol présente une faible hytérésis, pouvant devenir négligeable lorsque le nombre de cycles de chargement augmente (fig. 17)

Lorsque le rapport $\eta$ est supérieur à $\eta_{\mathrm{C}}$, l'hystérésis disparaît. Le chargement cyclique a pour effet une augmentation de volume traduisant le phénomène de dilatance du squelette granulaire (fig. 18).

5.2 Déformations sous des cycles de charges déviatoriques au triaxial conventionnel (trajet $(p, q)$ à pente 3$)$

Sur du sable de Fontainebleau à une contrainte de confinement de 0,2 $\mathrm{MPa}$, nous avons effectué à chaque niveau du déviateur appliqué, 20 cycles de chargement en contrainte déviatorique $\Delta \mathrm{q}=0,2 \mathrm{MPa}$.

La figure 19. a montre que:

1 - les déformations axiales $\epsilon_{t}^{p}$ irréversibles augmentent lorsque la contrainte déviatorique moyenne augmente :

II - pour un chargement en extension avec q négatif, le raidissement de la pente de la courbe $q, \epsilon$, est important :

III - le cycle d'hystérésis dans le diagramme $\left(q, \epsilon_{1}\right)$ se stabilise si le sol a été chargé juste précédemment à un niveau de contrainte plus élevé, (boucles 6 et 8 ).
La figure $19 . \mathrm{b}$ est particulièrement démonstrative et indique que :

1 - le comportement contractant du sol est obtenu lorsque la contrainte déviatorique moyenne est inférieure au seuil de l'état caractéristique :

II - le comportement dilatant au cours des cycles de chargement ne se manifeste que si la contrainte déviatorique moyenne est supérieure au seuil caractéristique;

III - si la contrainte maximale est plus faible que le seuil caractéristique, on obtient l'accommodation pour les cycles lorsque le nombre $\mathrm{N}$ de cycles augmente. Si le seuil caractéristique est dépassé, le rochet apparaît :

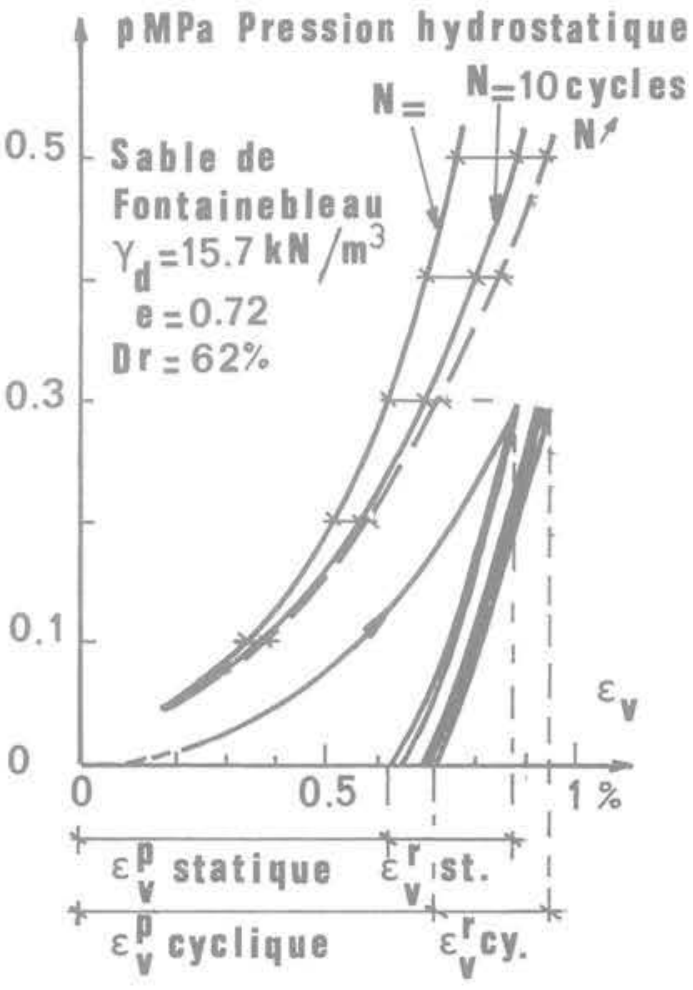

Fig. 16 Compression hydrostatique cyclique $(\eta=0)$
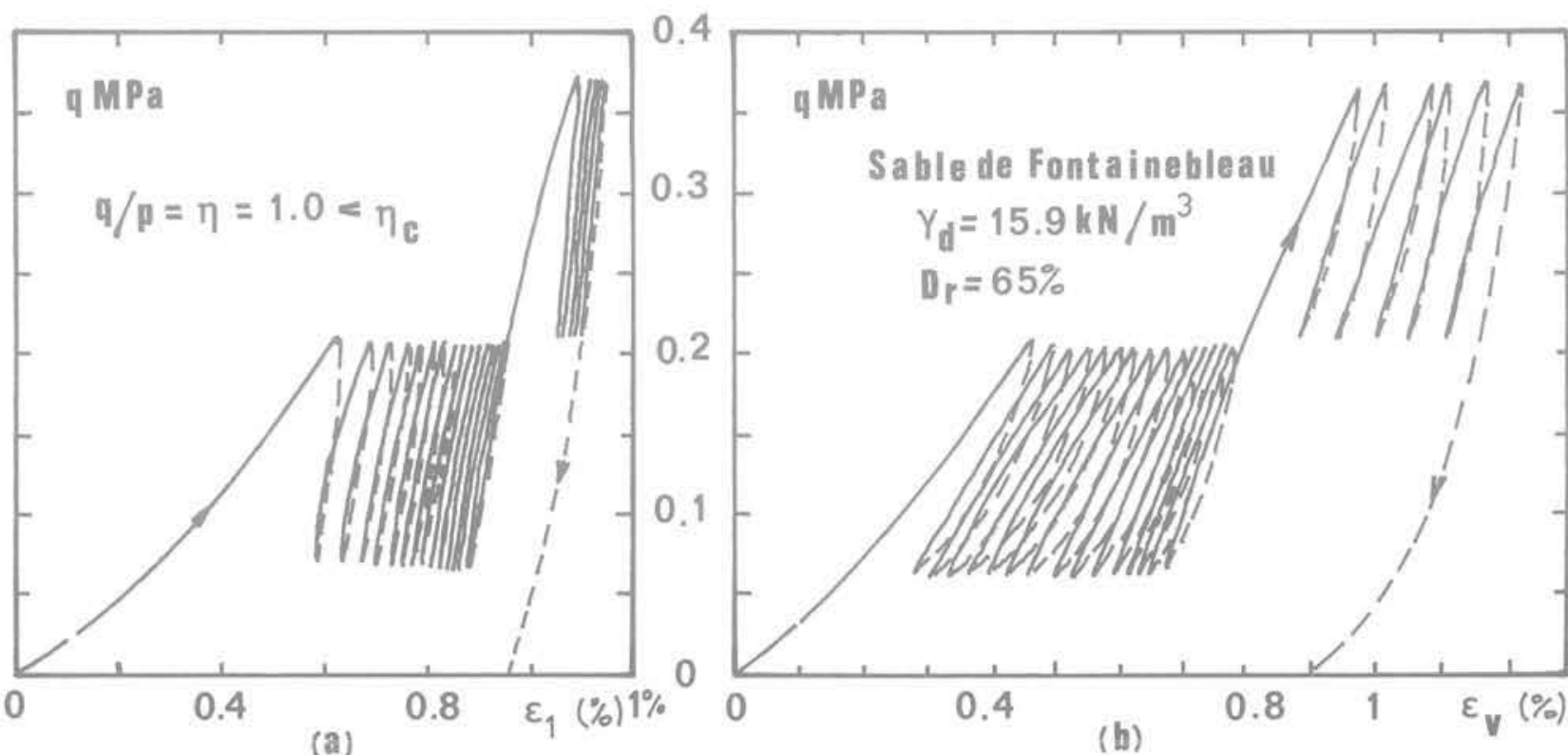

(b)

Fig. 17 Chargement radial à $\eta<\eta$ caractéristique 

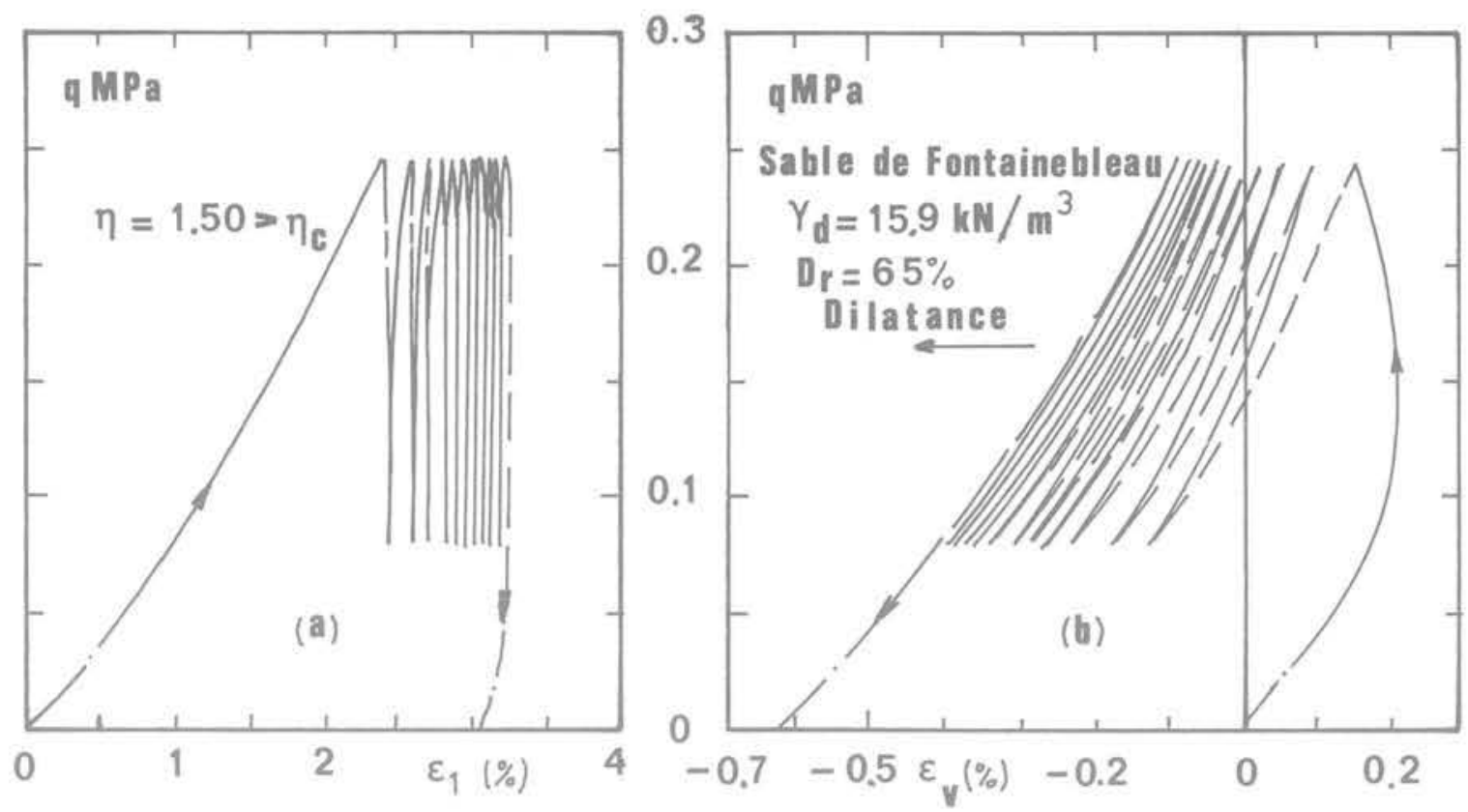

Fig. 18 Chargement radial à $\eta>\eta$ caractéristique
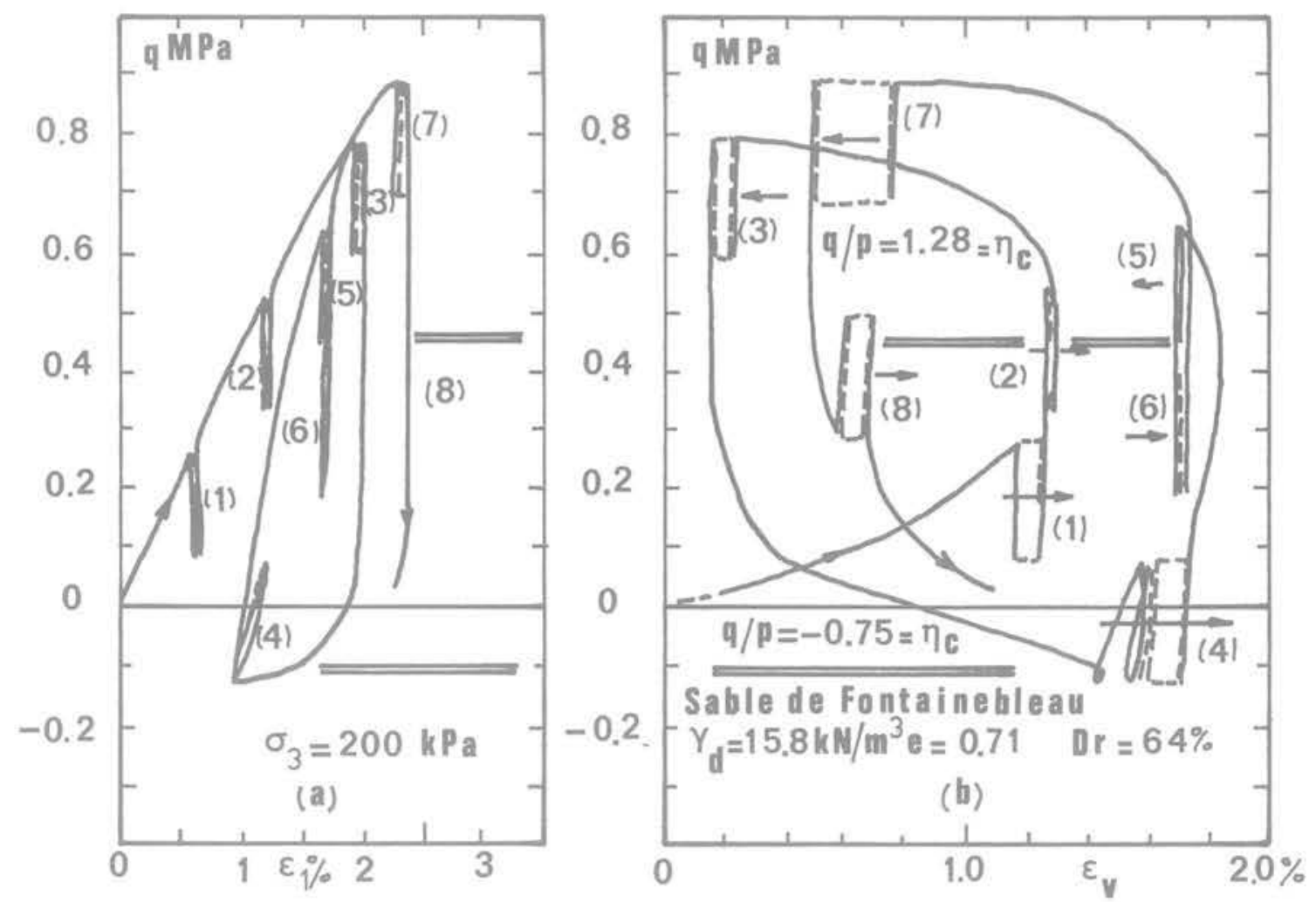

Fig. 19 Chargement cyclique à contrainte de confinement constante

IV - la contraction diminue lorsqu'on s'approche du seuil caractéristique; la dilatation augmente lorsqu'on s'en éloigne vers les fortes contraintes déviatoriques : (boucles 1,2 et 3 ) :

$V$ - l'effet contractant est plus significatifs pour un chargement en extension avec $q<0\left(\sigma_{1}<\sigma_{2}=\sigma_{3}\right)$; (boucle 4);

VI - l'effet dilatant est d'autant plus fort que le milieu devient plus dense.
5.3 Comportement contractant des sables denses Le phénomène de densification des sables denses peut être mis en évidence d'une façon simple par chargement cyclique avec dépassement des seuils caractéristiques en compression et extension triaxiales. Cette sollicitation à grande amplitude bénéficie d'un effacement de l'écrouissage lors de la phase dilatante qui désenchevêtre la structure granulaire du squelette solide. A chaque recharge, le mécanisme de serrage engendre de nouvelles déformations volumétriques irréversibles et se répète pour un nouveau matériau devenu chaque fois plus dense. 


\section{1 - Trajet triaxial conventionnel à confinement constant}

La figure 20.a montre un raidissement rapide d'un sable soumis à des cycles alternés de contrainte déviatorique. Cette densification est mise en évidence sur la figure 20.b oú l'on constate que l'indice des vides passe en quelques cycles de 0,720 à 0,682 .

Ce comportement indique l'influence déterminante de l'essai en extension, car le seuil caractéristique est rapidement atteint.

Ce résultat expérimental est en accord avec les essais de cisaillement direct de Youd (1972) sur du sable d'Ottawa; chaque cycle de cisaillement a présenté une séquence analogue de serrage-desserrage, et une déformation volumétrique irréversible finie s'accumule au cours des cycles pour donner une densité relative $D_{r} \approx 128 \%$ (norme ASTM D 3049-69), au bout de 10000 cycles de cisaillement d'amplitude $\pm 0,51 \mathrm{~mm}$.

\section{II - Trajet triaxial à contrainte moyenne $p$ constante}

La figure 21.a présente des boucles d'hystérésis $\left(q, \epsilon_{1}\right)$ qui se resserrent de plus en plus au cours des cycles. Sous ce type de chargement cyclique, le matériau semble tendre vers un état accomodé décrit par une boucle d'hystérésis $\left(q, \epsilon_{1}\right)$ stabilisée.

La figure 21.b traduit clairement le processus de densification avec ses boucles de dilatance aux niveaux caractéristiques en compression et en extension. La partie intermédiaire correspond à un serrage irréversible - entre deux séquences de réarrangement de la structure granulaire - qui comble progressivement les vides lacunaires existants.

III - Trajet circulaire à module de contraintes $(p, q)$ constant

Les figures 22.a et b confirment le phénomène de densification sous chargement cyclique de grande amplitude dépassant les seuils caractéristiques en compression et extension triaxiales. Quel que soit le trajet de charge, la forme des courbes est á peine modifiée, le mécan isme "boucle de dilatance suivi d'un serrage irréversible" reste parfaitement conservé au cours des cycles.

\subsection{Cisaillement drainé cyclique à déviateur q constant}

Les figures 23 indiquent clairement un processus de serrage progressif lorsque le matériau granulaire est soumis à un cisaillement drainé cyclique à déviateur q constant dans le domaine subcaractéristique $(\eta<\eta c)$ que ce soit en extension (a-b) ou en compression triaxiale (c-d et e-f). Le concept de l'état caractéristique se trouve donc encore une fois vérifié et conforté dans la proposition d'un domaine subcaractéristique contractant quel que soit le trajet suivi de la sollicitation cyclique.

\subsection{Liquéfaction du sable fin saturé en condition non drainée}

Les figures 24.a.b.c. décrivent le comportement d'un échantillon cylindrique de sable de Fontainebleau

$\left(\gamma_{C}=1,58 \cdot 10^{4} \mathrm{~N} / \mathrm{m}^{3} \mathrm{e}=0,710\right)$ saturé soumis à une pression de confinement $\sigma_{3}=0,12 \mathrm{MPa}$ et un déviateur cyclique $\Delta \mathrm{q}$ variant de $-0,01$ à $0,2 \mathrm{MPa}$ en condition non drainée et à vitesse de déformation axiale constante $\epsilon_{1}=6,83 \cdot 10^{-4} \mathrm{~s}^{-1}$. La liquéfaction du sable se manifeste pour $\mathrm{N}=34$ cycles par une augmentation brusque de la déformation axiale $\epsilon_{1}$ accompagnée d'une surpression interstitielle égalant presque la pression de confinement lorsque le déviateur est au voisinage de la valeur nulle.

L'instabilité du phénomène de liquéfaction est correctement interprétée par le concept de l'état caractéristique. Le point figuratif de la contrainte effective $\left(q, p^{\prime}\right)$ reste en effet à l'intérieur du domaine limité par les seuils caractéristiques en compression et en extension triaxiales. Dès que la contrainte effective atteint le seuil caractéristique en compression comme en extension, le point figuratif remonte la ligne caractéristique. La déformabilité devient de plus en plus grande lors de la décharge, entrainant un accroissement sensible de la pression interstitielle qui s'accumule au cours des cycles jusqu'à la ruine de l'échantillon. Le phénomène de liquéfaction totale n'est possible que si le chargement est alterné autour de l'état $q=0$.

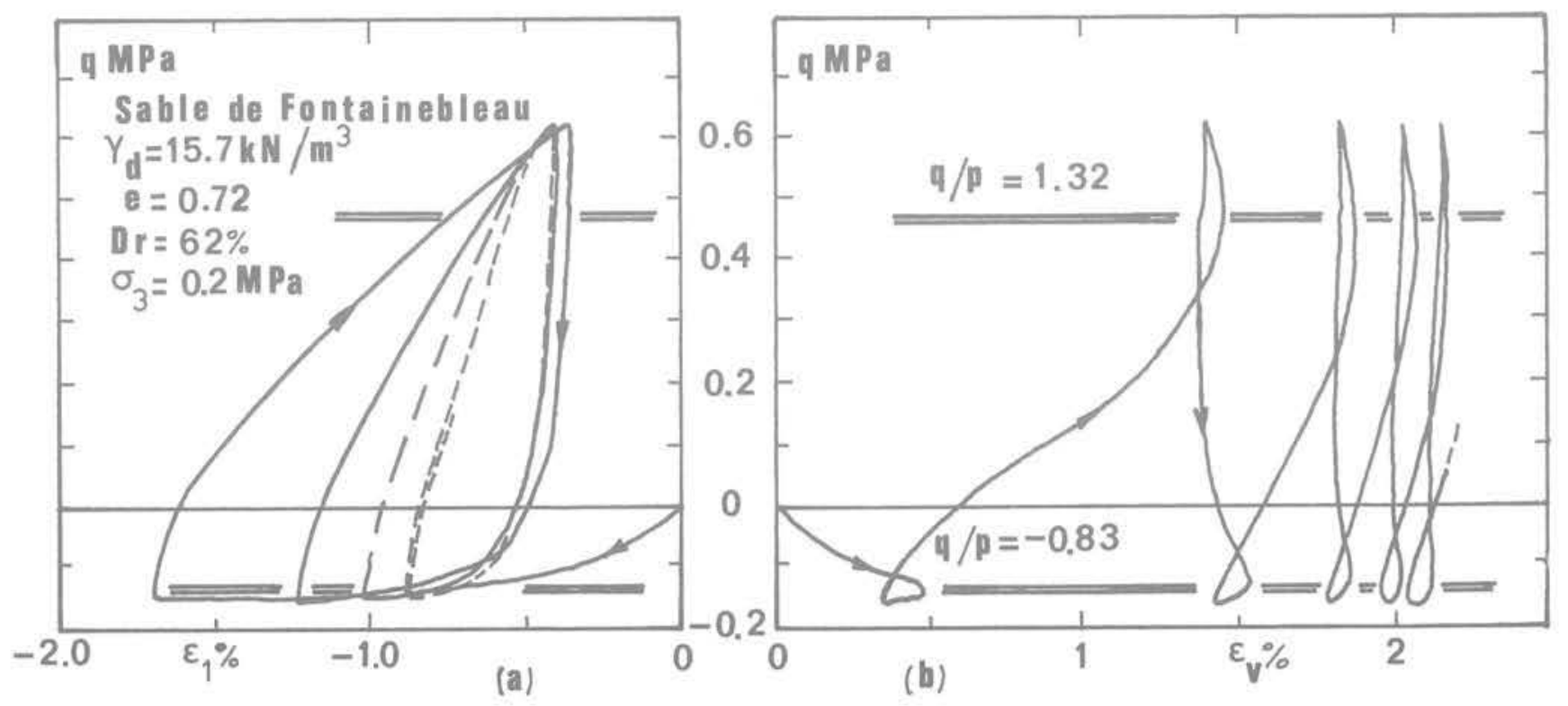

Fig. 20 Densification des sables denses à confinement constant 


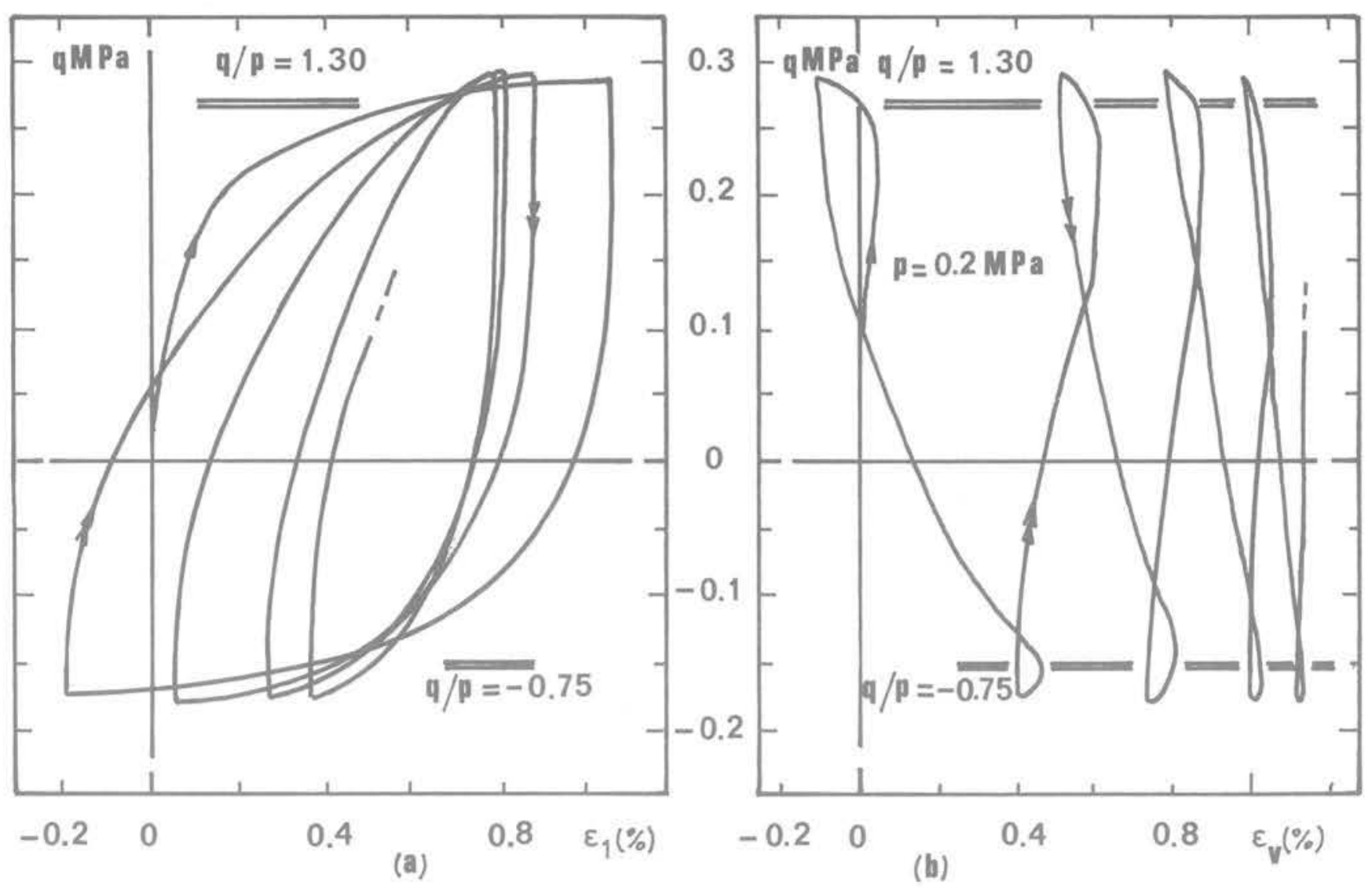

Fig. 21 Densification sous chargement de grande amplitude à $p=$ Constante
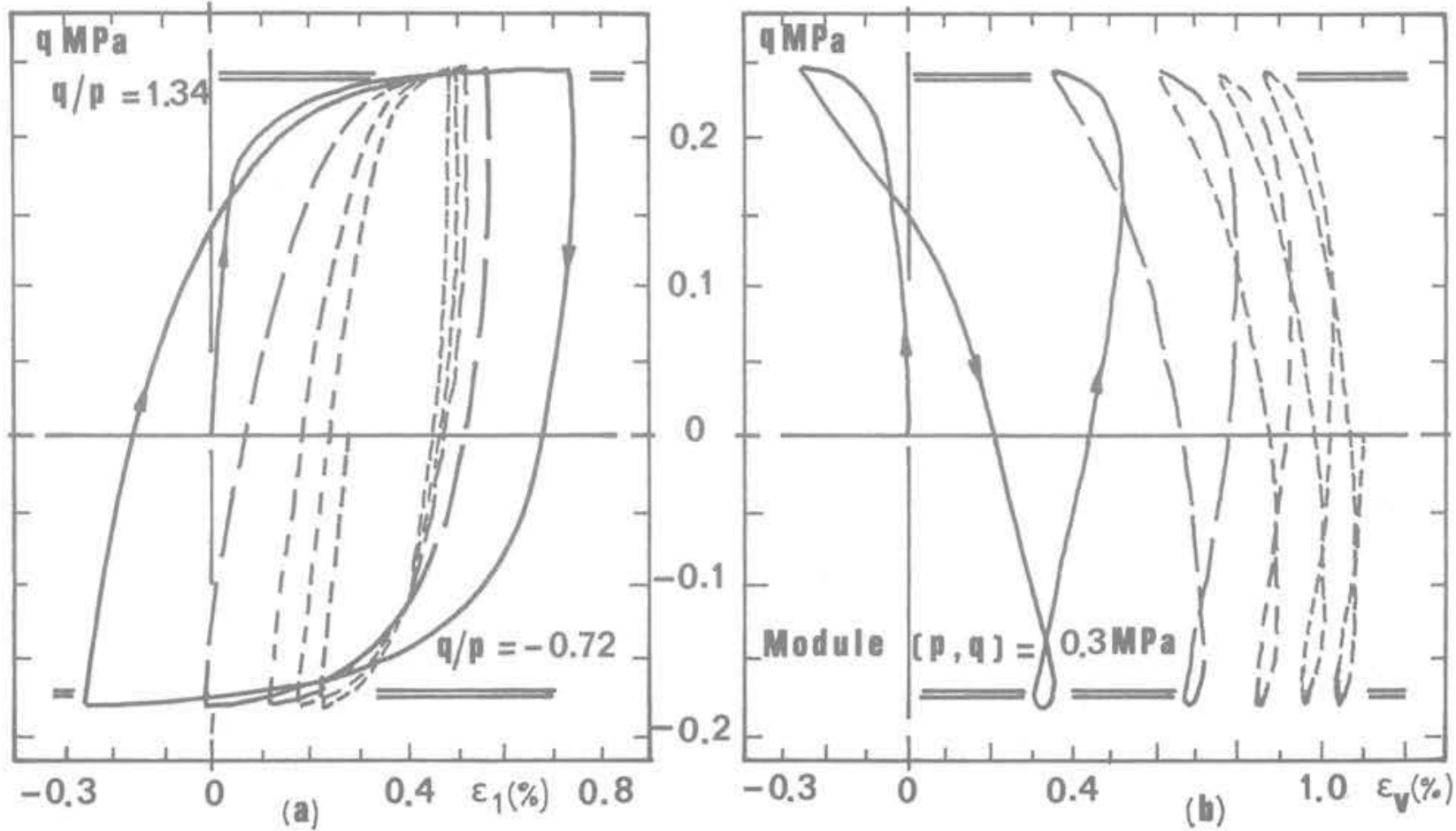

Fig. 22 Densification sous chargement cyclique de grande amplitude à trajet circulaire 


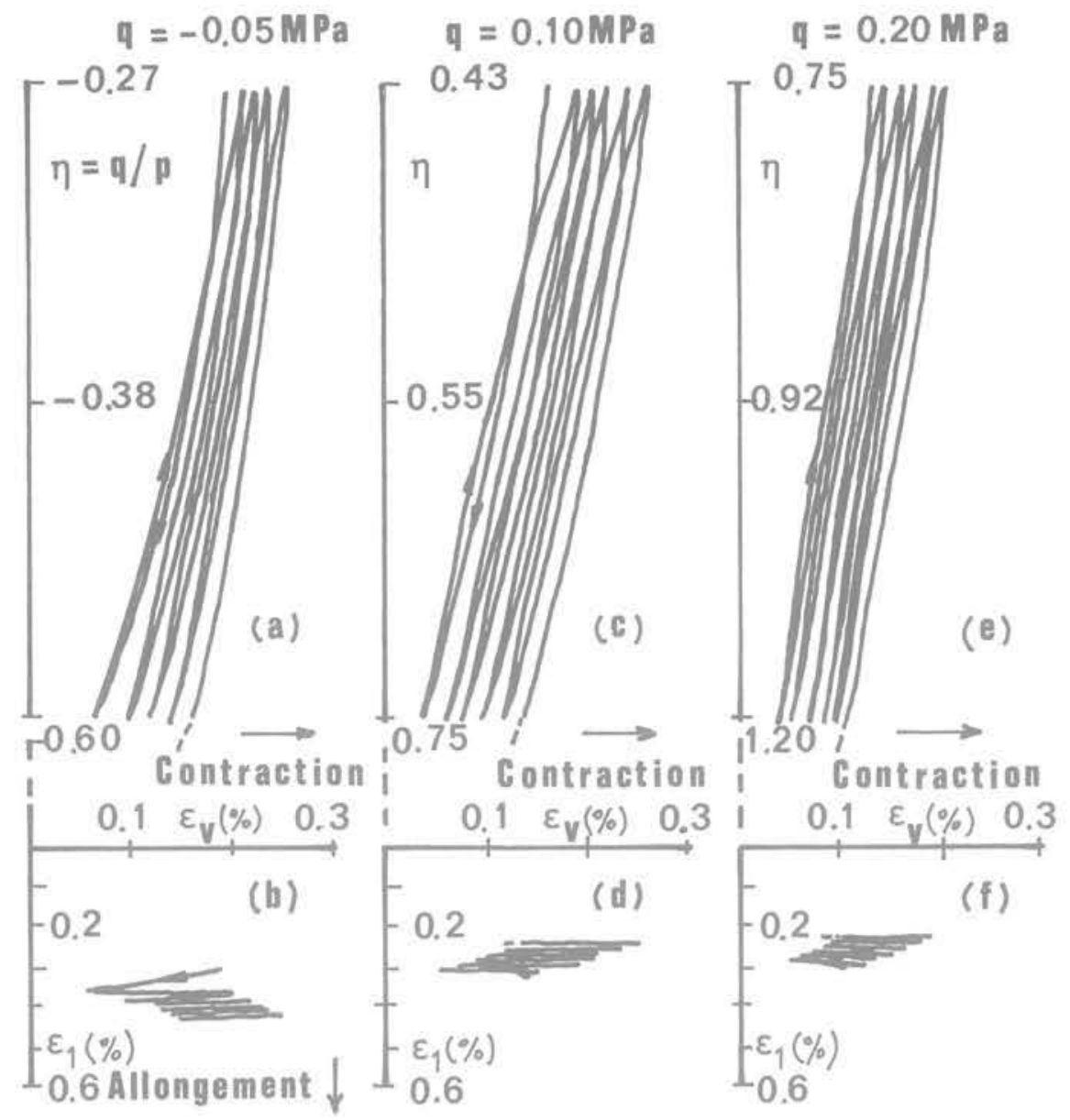

Fig. 23 Densification du sable de Fontainebleau sous chargement cyclique à déviateur $q=$ Constante
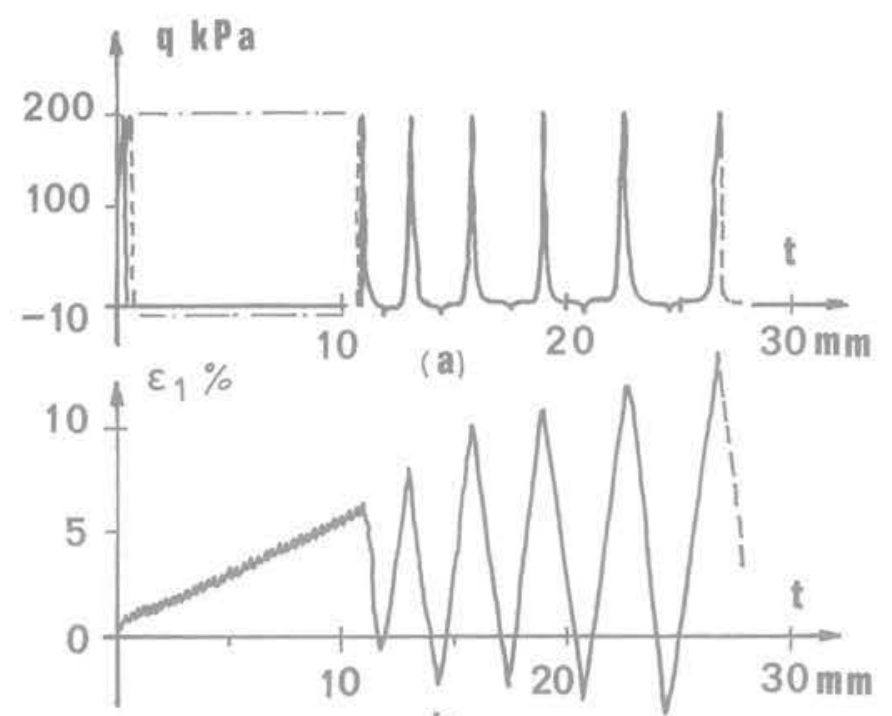

(b)

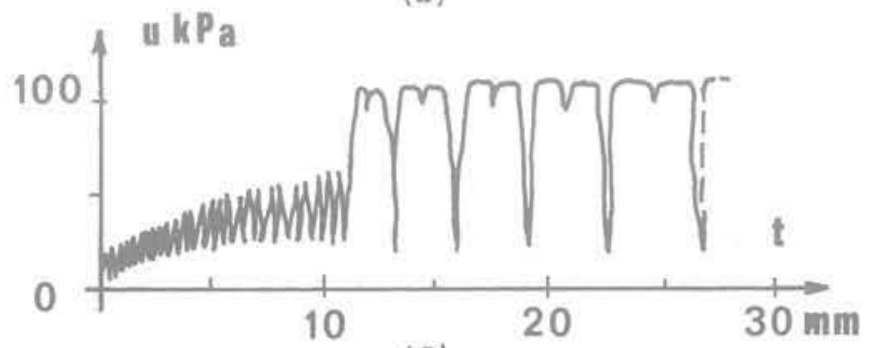

(C)

Fig. 24 Liquéfaction du sable de Fontainebleau $(e=0,72 ; D r=62 \%)$

\subsection{Mobilité cyclique et stabilisation}

Sur le trajet triaxial conventionnel, et sous un nombre finide cycles de déviateur q non nul, de signe constant, en condition non drainée, on peut constater un amollissement limité du sol dủ à une surpression interstitielle cyclique constante (mobilité cyclique) ou à un durcissement limité dans le cas d'une diminution de pression interstitielle cyclique constante (stabilisation).

La figure 25 résume les différents phénomènes rhéologiques rencontrés lors des essais triaxiaux conventionnels sur du sable en condition drainée et en condition non drainée.

\section{Conclusions}

6.1 Le paramètre essentiel dans l'étude du comportement rhéologique des sols est l'évolution de la déformation volumétrique au cours du chargement. L'angle $\varphi_{C}$ est un facteur intrinsèque caractérisant la capacité d'enchevêtrement du sol.

6.2 Le concept de l'état caractéristique mis en évidence et formulé de façon très simple sur un trajet de charge usuel de laboratoire s'est révélé vérifié et conforté par divers autres chemins de contraintes dans le plan $(p, q)$.Il peut être défini par un niveau distorsionnel $\beta$ s'accroîssant brusquement vers une forte valeur.

6.3 En condition non drainée ou à volume constant, le domaine subcaractéristique est un lieu géométrique pour le point figuratif de la contrainte effective. Dès que le trajet en contrainte effective atteint le seuil caractéristique, il remonte la ligne caractéristique. La longueur ainsi parcou- 


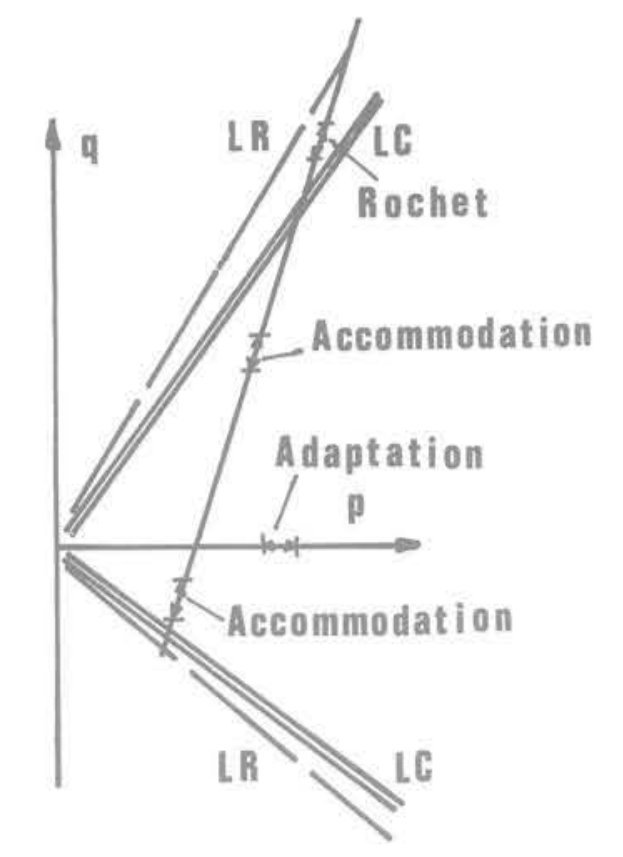

Sable sec ou en condition drainée

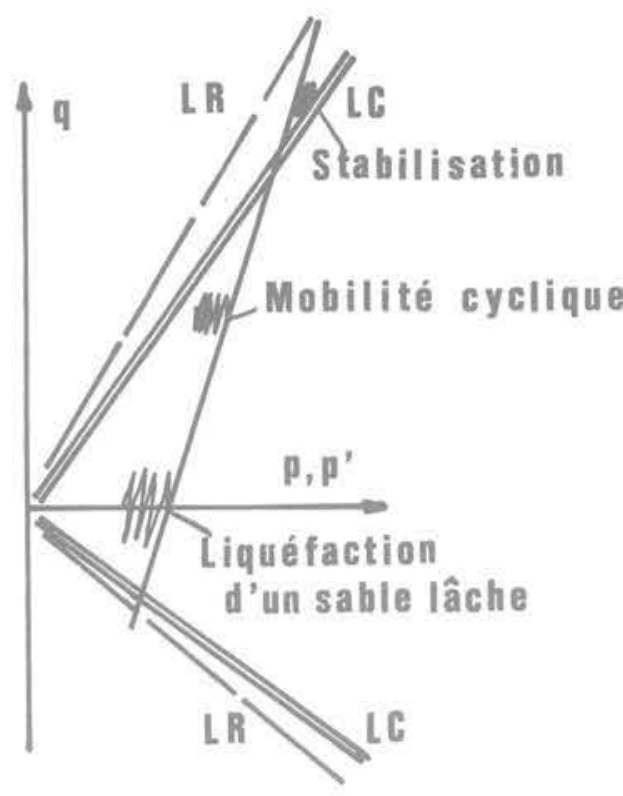

Sable en condition non drainée

Fig. 25 Différents phénomènes cycliques observés sur le trajet triaxial conventionnel

rue sur la ligne caractéristique détermine la perte de mèmoire de l'histoire antérieure lors de la décharge ou l'effacement de l'écrouissage. La recherche est associée à un nouvel état initial vierge.

6.4 Ce concept prend toute son importance dans le cas des chargements cycliques, car il permet de délimiter un domaine où l'effet des cycles quel que soit le chemin de contrainte est contractant. Un critère de liquéfiabilité en contrainte s'en déduit facilement : le point figuratif de la contrainte effective n'atteint l'origine que pour des chargements en contrainte totale alternée autour de $q=0$.

Au-delà, dans le domaine surcaractéristique limité supérieurement par rupture statique, l'effet des cycles est dilatant.

6.5 Les divers comportements cycliques des sols pulvérulents étudiés à l'appareil triaxial conventionnel sont aisément interprétés dans le cadre du concept de l'état caractéristique.

\section{Références Bibliographiques}

BOUTWELL G.P. Jr. (1968) - On the yield behaviour of cohesionless materials, Soil Mechanics, $n^{\circ} 7$, Duke University Thesis.

EL-SOHBY M.A., (1969) - Deformation of sands under constant stress ratios, 7 th ICSMFE, pp. 111-119, Mexico.

FLAVIGNY E., DARVE F., (1979) - Membrane penetration and its effect on pore pressure, Discussion ASCE GT2, vol. 105, Janvier 1979, pp. 115-117.
HABIB P., LUONG M.P., (1978) - Sols pulverulents sous chargements cycliques, Séminaire Matériaux et Structures sous Chargement Cyclique, 28-29 septembre 1978, Ecole Polytechnique, 91128 Palaiseau.

KIRKPATRICK W.M., (1961) - Discussion on Soil Properties and their Measurement, Proc. 5th Int. Conf. on Soil Mech. and Found. Eng., III, pp. 131-133, Paris.

KO H.Y.. SCOTT R.F. (1967) - Deformation of sand in hydrostatic compression, J. S.M.F. ASCE, vol. 93, SM3, pp. 137-156.

LUONG M.P., (1978) - Etat caractéristique du sol, C.R.Ac.Sc., Paris, t. 287, série B, 305.

LUONG M.P. (1978) - Comportements cycliques des sols pulvérulents, C.R.Ac.Sc., Paris, t. 287, série B, 313.

LUONG M.P., (1979) - Les phénomènes cycliques dans les sables, Journée de Rhéologie 1979, E.N.T.P.E., Vaulx-enVelin, 25 avril 1979.

LUONG M.P., (1979) - Aspects rhéologiques des sols sous sollicitations cycliques et transitoires. Séminaire Effets Cycliques et Transitoires dans les Structures, St-Rémy-lèsChevreuse, 23-25 octobre 1979.

LUONG M.P., (1980) - Stress-strain aspects of cohesionless soils under cyclic and transient loading, Int. Symp. Swansea, Wales, january 1980.

ROWE P.W., (1971) - Theoretical meaning and observed values of deformation for soils, Stress Strain Behaviour of Soils, Cambridge, march 1971, pp. 143-194, (ed. R.G.H. Parry).

SCHOFIELD A.N., WROTH C.P., (1968) - Critical State Soil Mechanics, Mc Graw Hill, London G.B.

TAYLOR D.W., (1948) - Fundamentals of Soil Mechanics, John Wiley, New-York.

YOUD T.L., (1972) - Compaction of Sands by Repeated Shear Straining, ASCE 98, SM7, pp. 709-725, july 1972. 
